\section{The Dynamics of the Hemo- static Parameters in Physio- logical Pregnancy and After Delivery}

\section{Andrey Pavlovich Momot ${ }^{1,2 *}$, Nadezhda Andreevna Semeno- va $^{2}$, Dmitry Evgenyevich Belozerov ${ }^{3}$, Dina Anatolyevna Trukhina $^{3}$ and Irina Yuryevna Kudinova ${ }^{3}$}

${ }^{1}$ Altai Branch, FSFI National Research Center for Hematology, Ministry of Healthcare of the Russian Federation, Barnaul, Altai Region, Russia

${ }^{2}$ Altai State Medical University, Barnaul, Altai Region, Russia

${ }^{3}$ Regional Clinical Hospital, Barnaul, Altai Region, Russia

\begin{abstract}
Nowadays hematologists and obstetrician-gynecologists have little data about the peculiarities of hemostatic parameters and the maintenance of hemostatic balance at different stages of physiological pregnancy. However, it is necessary to know the norms for the determination of pathologic deviations in the system and medical decision making. The article presents the dynamics of 50 hemostatic parameters in women in pregravid period, at different stages of physiological pregnancy, and 2-3 days after vaginal delivery studied in 301 Caucasian (white) women. The parameters that characterize different components of hemostatic system were taken into account-vascular-platelet, coagulant, anticoagulant, and Fibrinolytic. Thrombin generation test was used as an integrated method to study hemocoagulation.

According to the obtained results, some parameters were identified. These parameters could be an objective prerequisite for the prescription and monitoring the effects of antiaggregants and low molecular heparins during pregnancy with the aim of thromboprophylaxis. It is suggested that hyperproduction of Tissue Factor (TF) and thrombin (in relation to reference ranges) should play a possible role in initiation not only intravascular blood coagulation but also the end of pregnancy. It might be of importance in understanding the causes of preterm delivery. The described mechanism, preventing thrombus formation in late pregnancy, is associated with the enhancement of fibrinolytic reactions in blood circulation. This mechanism can lead to the degradation of soluble fibrin polymer (with the formation of D-dimers) before its conversion into gel or fibrin clot (fibrin insolubile). The obtained data could be useful in future to identify pregnant women at risk dealing with thrombosis,
\end{abstract}

${ }^{*}$ Corresponding author: Andrey Pavlovich Momot, Altai Branch, FSFI National Research Center of Hematology, Ministry of Healthcare of Russian Federation Barnaul, Altai Region, Russia, Tel: +7 3852689800; E-mail: xyzan@yandex.ru

Citation: Momot AP, Semenova NA, Belozerov DE, Trukhina DA, Kudinova IY (2016) The Dynamics of the Hemostatic Parameters in Physiological Pregnancy and After Delivery. J Hematol Blood Transfus Disord 3: 005

Received: December 02, 2015; Accepted: February 12, 2016; Published: February 26, 2016 bleeding and obstetrics complications during pregnancy and delivery.

Keywords: Blood coagulation markers; Coagulation factors; Fibrinolysis; Hemostatic system; Physiological anticoagulants; Pregnancy; Thrombin generation

\section{Abbreviations}

ADAMTS-13: Metalloproteinase which cleaves von Willebrand factor ("a disintegrin and metalloproteinase with a thrombospondin type 1 motif, member 13")

ADP: Adenosine Diphosphate

APTT : Activated Partial Thromboplastin Time

AT III: Antithrombin III

$\boldsymbol{\alpha}_{2}$-AP: $\boldsymbol{\alpha}_{2}$-Antiplasmin

ETP: Endogenous Thrombin Potential

FVIIa: Activated Factor VII

MTHFR : Methylene Tetrahydrofolate Reductase

PAI-1: Plasminogen Activator Inhibitor 1

PAI-2: Plasminogen Activator Inhibitor 2

PF4: Platelet Factor 4

PT: Prothrombin Time

RCoA: Ristocetin Cofactor Activity von Willebrand Factor

TAFI: Thrombin Activated Fibrinolysis Inhibitor

TF: Tissue Factor

TFPI: Tissue Factor Pathway Inhibitor

ttPeak: Time to Reach Peak Thrombin

t-PA: Tissue-type Plasminogen Activator

TTP: Thrombotic Thrombocytopenic Purpura

vWF Ag: von Willebrand Factor Antigen

u-PA: urokinase-Type Plasminogen Activator

\section{Introduction}

Pregnancy, delivery and postpartum period are closely connected with high morbidity and mortality rates $[1,2]$. The morbidity and mortality are associated with deviations from the physiological activation of blood coagulation norm progressing through gestation that could reduce blood loss during delivery. On the one hand, pregnancy and early postpartum period are at high risk of venous thrombosis (4-50 times higher than in nonpregnant women $[3,4]$ and, on the other hand, uterine hemorrhage is typical of all gestation periods but severe obstetric hemorrhage are dangerous complications for lives [5]. The facts are of great medical attention to this period of women's lives.

Despite a large number of scientific publications devoted to the peculiarities of changes in hemocoagulation during pregnancy [6-9], there are unsolved problems today. The problems are that in practice the laboratory parameters of the hemostatic system in pregnant women are generally related to the intervals of allowed values of the panel of hemostatic parameters determined for nonpregnant women that do not allow making grounded clinical decisions [9]. The differences in the diagnostic test technologies make additional difficulties in the result interpretation. So, the study of the allowed values of hemostatic parameters during pregnancy and after delivery in relation to the specific equipment and test systems is of great 
interest since these data can be useful for the differential diagnostics of bleeding causes, as well as for the identification of patients needed in thromboprophylaxis.

Moreover, simultaneous identification of a lot of parameters, characterizing coagulation, anticoagulant and fibrinolytic activity of blood could allow obtaining the important information about dynamically changing hemostatic balance during pregnancy, which is normally accompanied by neither bleedings nor thromboses. The study of these parameters is expected to explain the causes of 3-4 fold increase in D-dimers level in late physiological pregnancy, to select objective factors for initiation and cessation of heparinprophylaxis and to solve other problems associated with pregnancy complications.

The aim of this study is to determine the allowed values and the dynamics of changes of a wide range of hemostatic parameters at different stages of physiological pregnancy and the first few days after delivery.

\section{Methods}

\section{Study population}

Within a prospective study, there were data obtained from 301 Caucasian (white) women who provided their informed consent. The study was approved by the local research Ethics Committee of the Altai State Medical University (Protocol 14, from 11.12.1013.). Based on the results of clinical and laboratory examinations, there was an advisory by obstetrician-gynecologist, therapist, and hematologist and after that it was decided whether to enroll women in different stages of pregnancy in the present study. According to the inclusion and exclusion criteria, all the women enrolled in the study were basically examined 1-3 times during the period of examination.

The inclusion criteria were singleton pregnant, multigravida women from 18 to 35 , and women having spontaneous labor Women over 35 years old with the history of obstetric and gynecological disorders, personal hemorrhage or thrombosis, administration of combined oral contraception for at least 3 months before pregnancy, used assisted reproductive technologies, operative delivery were excluded from the present study. Besides, exclusion criteria included extragenital pathology including diabetes mellitus, antiphospholipid syndrome, autoimmune states, endocrine disorders, the presence of clinical signs of mesenchymal dysplasia, heart valve or vessels prostheses, acute pyelonephritis, acute cystitis, malignant neoplasms, human immunodeficiency virus carrier state, viral hepatitis type B or C carrier state. Women with drug administration during pregnancy affecting the hemostatic system lowering platelet aggregation (nonsteroidal anti-inflammatory drug, anticoagulants) were also excluded. Deviations in the results of laboratory examinations: homocysteine level in blood serum (before pregnancy) more than $15 \mu \mathrm{mol}$, hemoglobin more than $140 \mathrm{~g} / \mathrm{l}$ in nonpregnant women, platelet count less than 150 or more than $450 \times 10^{9} / 1$, white blood count more than $12 \times 10^{9} / 1$; factor $V$ Leiden mutation carrier state $(1691 \mathrm{G}>\mathrm{A})$ and/or FII mutation carrier state $(20210 \mathrm{G}>\mathrm{A})$, as well as rare homozygous Methylene Tetrahydrofolate Reductase (MTHFR) $(677 \mathrm{C}>\mathrm{T})$ were also considered as exclusion criteria.

So, sample size at different stages of the study (in nonpregnant state, at different stages of pregnancy, and after delivery) comprises women who matched the above mentioned criteria. The following endpoints were selected to study hemostatic system, taking into account trophoblast invasion waves and reflective "critical" stages of pregnancy: pregravid period, 6-8 weeks, 12-13 weeks, 22-24 weeks,
34-36 weeks and 2-3 days after delivery. Studies in pregravid period were carried out mainly among primigravida women (81.5\%).

\section{Blood collection}

Venous blood collection was carried out from the cubital vein to the test tubes Vacuette with buffer solution of sodium citrate in a ratio of 9:1 (9NC Coagulation sodium citrate $3.2 \%$ ). Blood was centrifuged at $1400 \mathrm{~g}$ for $15 \mathrm{~min}$ at room temperature resulted in platelet poor plasma. To study platelet function, platelet enriched plasma was obtained by blood centrifugation at $160 \mathrm{~g}$ for $7 \mathrm{~min}$ under the same temperature conditions. Plasma samples were studied during the first two hours. Plasma was stored in $1.5 \mathrm{ml}$ Eppendorf tubes filled to $3 / 4$ at temperature $-40^{\circ} \mathrm{C}$ in the time interval from one day to one month in MDF-192 Ultra low temperature freezer (Sanyo) to conduct enzyme immune analysis or endogenous thrombin potential assessment by thrombin generation test. To determine homocysteine level in blood serum, vacuum tubes Improvacuter (Lind-Vac) were used without filler. Platelet counts were determined in stabilized EDTA blood received with vacuum tubes Improvacuter (Lind-Vac) with dissolved stabilizer-EDTA K3.

\section{Laboratory assays}

In the study the following equipment was used: Hematology Analyzer MEK-7222 J/K [1] (Nihon Kohden); optical aggregometer AggRAM [2] (Helena Laboratories); semi-automated coagulometers Helena C-1 [3] (Helena Laboratories) and Start 4 [4] (Stago); automated coagulometers-STA Compact [5] (Stago) and Sysmex CA-1500 [6] (Sysmex Corporation); microplate fluorometer Fluoroskan Ascent [7] (Thermo Fisher SCIENTIFIC) with software «Thrombinoscope 3.0.0.26»; immunochemical analyzer-IMMULITE 2000 [8] (Siemens Healthcare Diagnostics); photometers-spectrophotometer Photometr 5010 v5+ [9] (Robert Riele GmbH \& Co KG) and microplate reader Bio-Rad 680 [10] (Bio-Rad Laboratories); express analyzer (reflectometer) NycoCard Rider II [11] (Axis Shield).

Note: The square brackets mark the equipment used for the implementation of various methods for diagnostics of hemostatic system (Table 1).

In the study test systems produced by the following companies were used: «AssayPro», «St. Charles, MO», «Axis-Shield», «Siemens», «BCM Diagnostics», «Biomedica», «Helena Bioscience», «Sekisui Diagnostics» («American Diagnostica»), «Technoclone» and «Thrombinoscope BV». The analysis was performed in accordance with the instructions to the kits of reagents and recommendations of known guidelines in the field of laboratory diagnostics of hemostatic disorders $[10,11]$.

Coagulation of the studied blood plasma in the assessment of the parameters of thrombin generation [12] was carried out in the presence of $5 \mathrm{pmol}$ of tissue factor and $4 \mu \mathrm{mol}$ of phospholipid (PPP-Reagent 5pM, Thrombin Calibrator, FluCa-Kit). Thrombin generation was registered by measurement of fluorogenic substance signal (Z-Gly-Gly-Arg-AMC). The studies of each blood plasma sample were performed in two parallel settings. For genotyping Factor V Leiden mutation, Factor II mutation, and MTHFR, method of real time polymerase chain reaction with TagMan probes was used.

Freshly obtained pooled platelet poor plasma from 5-10 healthy women from 18 to 35 years was used as control normal plasma to calculate the ratio and Normalized Ratio (NR) in the assessment of the results of methods marked in the table under № 1.4.1, 2.1-2.5, 6.2. 
Citation: Momot AP, Semenova NA, Belozerov DE, Trukhina DA, Kudinova IY (2016) The Dynamics of the Hemostatic Parameters in Physiological Pregnancy and After Delivery. J Hematol Blood Transfus Disord 3: 005

- Page 3 of $18 \cdot$

\begin{tabular}{|c|c|c|c|c|c|c|c|}
\hline $\begin{array}{l}\text { Method, Unit } \\
\text { (1) }\end{array}$ & $\begin{array}{l}\text { Reagent / manufacture } \\
\text { (2) }\end{array}$ & $\begin{array}{l}\text { Pregravid } \\
\text { period } \\
\text { (3) }\end{array}$ & $\begin{array}{l}\text { 6-8 weeks } \\
\text { (4) }\end{array}$ & $\begin{array}{l}\text { 12-13 weeks } \\
(5)\end{array}$ & $\begin{array}{l}\text { 22-24 weeks } \\
\text { (6) }\end{array}$ & $\begin{array}{l}\text { 34-36 weeks } \\
(7)\end{array}$ & $\begin{array}{l}\text { 2-3 days after } \\
\text { delivery } \\
\text { (8) }\end{array}$ \\
\hline \multicolumn{8}{|c|}{ 1. Vascular-thrombotic hemostasis and markers for endotheliosis } \\
\hline $\begin{array}{l}\text { 1.1. Platelet count in } \\
\text { blood, } \times 109 /[11]\end{array}$ & - & $\begin{array}{c}239[162-310] \\
n=48\end{array}$ & $\begin{array}{c}233[141-334] \\
n=50\end{array}$ & $\begin{array}{c}243[152-323] \\
n=41\end{array}$ & $\begin{array}{c}246\left[\begin{array}{c}{[135-393]} \\
n=5\end{array}\right. \\
n\end{array}$ & $\begin{array}{c}222[135-348] \\
n=38\end{array}$ & $\begin{array}{c}227[160-465] \\
n=31\end{array}$ \\
\hline $\begin{array}{l}\text { 1.2. Platelet Factor 4, } \\
\text { level, } \mathrm{ng} / \mathrm{ml}[11]\end{array}$ & $\begin{array}{l}\text { IMUCLONE Platelet Factor } 4 \\
\text { ELISA kit/Sekisui Diagnostics } \\
\text { (American Diagnostica) }\end{array}$ & $\begin{array}{c}30[21-39] \\
n=48\end{array}$ & $\begin{array}{c}26[22-38] \\
n=49\end{array}$ & $\begin{array}{c}27[21-41] \\
n=40\end{array}$ & $\begin{array}{c}28[20-36] \\
n=55\end{array}$ & $\begin{array}{c}27[20-41] \\
n=38\end{array}$ & $\begin{array}{c}27[22-37] \\
\mathrm{n}=47\end{array}$ \\
\hline \multicolumn{8}{|c|}{ 1.3. Platelet aggregation, $\%$ aggregation with agonists (taken at the following final concentration in the aggregometer cuvette) } \\
\hline $\begin{array}{l}\text { 1.3.1. Arachidonic acid } \\
(0,5 \mathrm{mmol} / /)[2]\end{array}$ & $\begin{array}{c}\text { Arachidonic Acid/Helena } \\
\text { Bioscience }\end{array}$ & $\begin{array}{c}84[67-97] \\
n=36\end{array}$ & $\begin{array}{c}85[70-88] \\
n=45\end{array}$ & $\begin{array}{c}86[73-103] \\
n=40\end{array}$ & $\begin{array}{c}84[69-93] \\
n=56\end{array}$ & $\begin{array}{c}81[65-102] \\
n=39\end{array}$ & $\begin{array}{c}82[68-93] \\
n=46\end{array}$ \\
\hline $\begin{array}{l}\text { 1.3.2. ADP }(0,1 \mu \mathrm{mo} / / \mathrm{l}) \\
{[2]}\end{array}$ & ADP/Helena Bioscience & $\begin{array}{c}10[4-23] \\
{[11.2 \pm 0.9]} \\
\mathrm{n}=42\end{array}$ & $\begin{array}{c}13[3-37] \\
{[13.6 \pm 1.2]} \\
n=42\end{array}$ & $\begin{array}{c}19[7-69] \\
{[25.9 \pm 3.5]} \\
n=36\end{array}$ & $\begin{array}{c}64[10-86] \\
{[53.6 \pm 3.6]} \\
n=51\end{array}$ & $\begin{array}{c}47[10-92] \\
{[45.9 \pm 5.3]} \\
\mathrm{n}=33\end{array}$ & $\begin{array}{c}38[12-78] \\
{[40.9 \pm 3.6]} \\
\mathrm{n}=43\end{array}$ \\
\hline $\begin{array}{l}\text { 1.3.3. ADP }(2,0 \mu \mathrm{mo} / / \mathrm{l}) \\
{[2]}\end{array}$ & ADP/Helena Bioscience & $\begin{array}{c}81[60-95] \\
{[79.9 \pm 1.7]} \\
\mathrm{n}=42\end{array}$ & $\begin{array}{c}83[73-99] \\
{[83.5 \pm 1.3]} \\
\mathrm{n}=48\end{array}$ & $\begin{array}{c}82[69-100] \\
{[83.4 \pm 1.6]} \\
n=38\end{array}$ & $\begin{array}{c}82[66-93] \\
{[81.3 \pm 0.9]} \\
n=54\end{array}$ & $\begin{array}{c}79[64-99] \\
{[80.6 \pm 1.7]} \\
n=36\end{array}$ & $\begin{array}{c}79[62-93] \\
{[79.5 \pm 1.1]} \\
\mathrm{n}=48\end{array}$ \\
\hline $\begin{array}{l}\text { 1.3.4. ADP (10,0 } \\
\mu \mathrm{mol} / \mathrm{l})[2]\end{array}$ & ADP/Helena Bioscience & $\begin{array}{c}82[58-93] \\
n=44\end{array}$ & $\begin{array}{c}84[68-94] \\
n=45\end{array}$ & $\begin{array}{c}86[70-97] \\
n=38\end{array}$ & $\begin{array}{c}84[71-91] \\
n=44\end{array}$ & $\begin{array}{c}81[62-103] \\
n=36\end{array}$ & $\begin{array}{c}82[58-94] \\
n=48\end{array}$ \\
\hline $\begin{array}{l}\text { 1.3.5. Adrenalin (5 } \\
\mu \mathrm{mol} / / \mathrm{l})[2]\end{array}$ & Epinephrine/Helena Bioscience & $\begin{array}{c}81[60-95] \\
n=42\end{array}$ & $\begin{array}{c}83[73-99] \\
n=48\end{array}$ & $\begin{array}{c}82[69-100] \\
n=38\end{array}$ & $\begin{array}{c}82[66-93] \\
n=54\end{array}$ & $\begin{array}{c}79[64-99] \\
n=36\end{array}$ & $\begin{array}{c}79[62-93] \\
n=48\end{array}$ \\
\hline $\begin{array}{l}\text { 1.3.6. Collagen }(10 \mu \mathrm{g} / \\
\mathrm{ml})[2]\end{array}$ & Collagen/Helena Bioscience & $\begin{array}{c}83[69-93] \\
n=44\end{array}$ & $84[72-94] n=50$ & $\begin{array}{c}84[70-97] \\
\mathrm{n}=40\end{array}$ & $\begin{array}{c}84[74-93] \\
n=54\end{array}$ & $\begin{array}{c}82[66-97] \\
n=37\end{array}$ & $\begin{array}{c}83[72-91] \\
n=48\end{array}$ \\
\hline $\begin{array}{l}\text { 1.3.7. Ristomycin }(1,5 \\
\mathrm{mg} / \mathrm{ml})[2]\end{array}$ & Ristocetin/Helena Bioscience & $\begin{array}{c}79[64-92] \\
n=42\end{array}$ & $\begin{array}{c}80[68-96] \\
n=46\end{array}$ & $\begin{array}{c}79[69-93] \\
n=39\end{array}$ & $\begin{array}{c}80[69-92] \\
n=54\end{array}$ & $\begin{array}{c}78[56-97] \\
n=36\end{array}$ & $\begin{array}{c}77[60-88] \\
n=48\end{array}$ \\
\hline \multicolumn{8}{|c|}{ 1.4. von Willebrand factor and metalloproteinase ADAMTS-13 } \\
\hline $\begin{array}{l}\text { 1.4.1. Ristocetin } \\
\text { cofactor activity, \% } \\
\text { aggregation [2] }\end{array}$ & $\begin{array}{l}\text { Ristocetin Cofactor Kit/Helena } \\
\text { Bioscience }\end{array}$ & $\begin{array}{c}95[54-152] \\
\mathrm{n}=40\end{array}$ & $\begin{array}{c}87[64-129] \\
n=47\end{array}$ & $\begin{array}{c}97[68-160] \\
n=40\end{array}$ & $\begin{array}{c}112[70-178] \\
n=45\end{array}$ & $\begin{array}{c}134\left[\begin{array}{c}75-167] \\
n=35\end{array}\right. \\
\text { n }\end{array}$ & $\begin{array}{c}139[89-179] \\
n=46\end{array}$ \\
\hline $\begin{array}{l}\text { 1.4.2. von Willebrand } \\
\text { factor in plasma, level, } \\
\%[10]\end{array}$ & $\begin{array}{l}\text { TECHNOZYM vWF:Ag ELISA/ } \\
\text { Technoclone GmbH' }\end{array}$ & $\begin{array}{c}106[70-154] \\
n=44\end{array}$ & $\begin{array}{c}119[79-182] \\
\mathrm{n}=47\end{array}$ & $\begin{array}{c}121[98-186] \\
n=36\end{array}$ & $\begin{array}{c}142[99-203] \\
n=51\end{array}$ & $\begin{array}{c}148\left[\begin{array}{c}105-208] \\
n=33\end{array}\right. \\
\text { n }\end{array}$ & $\begin{array}{c}165[95-223] \\
n=40\end{array}$ \\
\hline $\begin{array}{l}\text { 1.4.3. von Willebrand } \\
\text { factor in plasma, level, } \\
\%[6]\end{array}$ & vWF Ag/Siemens & $\begin{array}{c}102[62-157] \\
n=50\end{array}$ & $\begin{array}{c}124[71-222] \\
\mathrm{n}=48\end{array}$ & $\underset{\mathrm{n}=41}{159[79-260]}$ & $\begin{array}{c}190[104-260] \\
n=52\end{array}$ & 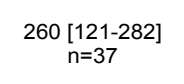 & $\begin{array}{c}260[170-291] \\
\mathrm{n}=48\end{array}$ \\
\hline $\begin{array}{l}\text { 1.4.4. Metallopro- } \\
\text { teinase ADAMTS-13, } \\
\text { activity, \% [10] }\end{array}$ & $\begin{array}{l}\text { TECHNOZYM ADAMTS-13 } \\
\text { Activity ELISA/Technoclone } \\
\text { GmbH }\end{array}$ & $\begin{array}{l}91[50-142] \\
n=51\end{array}$ & $\begin{array}{l}95[45-152] \\
n=50\end{array}$ & $\begin{array}{c}84[50-126] \\
n=42\end{array}$ & $\begin{array}{c}67[32-92] \\
n=57\end{array}$ & $\begin{array}{c}55[29-111] \\
n=38\end{array}$ & $\begin{array}{c}58[40-150] \\
\mathrm{n}=47\end{array}$ \\
\hline $\begin{array}{c}\text { 1.4.5. Metalloprotein- } \\
\text { ase ADAMTS-13, level, } \\
\mu \mathrm{g} / \mathrm{ml}[10]\end{array}$ & $\begin{array}{c}\text { TECHNOZYM ADAMTS-13 } \\
\text { Antigen ELISA/Technoclone } \\
\text { GmbH }\end{array}$ & $\begin{array}{c}1.64[0.89-2.88] \\
n=51\end{array}$ & $\begin{array}{c}1.71[1.04-3.04] \\
n=50\end{array}$ & $\begin{array}{c}1.65[1.09-2.54] \\
n=43\end{array}$ & $\begin{array}{c}1.17[0.79-1.82] \\
n=57\end{array}$ & $\begin{array}{c}1.12[0.65-1.76] \\
n=38\end{array}$ & $\begin{array}{c}1.02[0.70-2.24] \\
n=46\end{array}$ \\
\hline $\begin{array}{l}\text { 1.5. Endothelin -1, } \\
\text { level, fmol/ml [10] }\end{array}$ & ENDOTHELIN (1-21)/Biomedica & $\begin{array}{c}0.39[0.10-1.31] \\
n=51\end{array}$ & $\begin{array}{c}0.44[0.06-1.86] \\
n=47\end{array}$ & $\begin{array}{c}0.29[0.06-1.60] \\
n=40\end{array}$ & $\begin{array}{c}0.33[0.05-1.54] \\
n=50\end{array}$ & $\begin{array}{c}0.3[0.07-1.68] \\
n=37\end{array}$ & $\begin{array}{c}0.31[0.05-1.24] \\
n=44\end{array}$ \\
\hline $\begin{array}{l}\text { 1.6. Homocysteine, } \\
\mu \mathrm{mol} / /[8]\end{array}$ & $\begin{array}{l}\text { IMMULITE } 2000 \text { Homocysteine/ } \\
\text { Siemens }\end{array}$ & $\begin{array}{c}9.8[5.8-13.4] \\
n=50\end{array}$ & $\begin{array}{c}6.8[5.3-12.7] \\
n=28\end{array}$ & $\begin{array}{c}6.3[4.2-9.8] \\
\mathrm{n}=29\end{array}$ & $\begin{array}{c}5.4[3.0-8.4] \\
n=47\end{array}$ & $\begin{array}{c}5.9[4.2-8.9] \\
\mathrm{n}=33\end{array}$ & $\begin{array}{c}9.1[5.9-18.7] \\
n=48\end{array}$ \\
\hline \multicolumn{8}{|c|}{ 2. Screening coagulation tests } \\
\hline 2.1. APTT, sec, ratio [5] & Pathromtin SL/Siemens & $\begin{array}{c}1.09[0.92-1.24] \\
n=48\end{array}$ & $\begin{array}{c}1.04[0.86-1.25] \\
n=50\end{array}$ & $\begin{array}{c}0.97[0.88-1.14] \\
n=42\end{array}$ & $\begin{array}{c}0.96[0.83-1.14] \\
n=55\end{array}$ & $\begin{array}{c}0.95[0.85-1.15] \\
n=40\end{array}$ & $\begin{array}{c}0.93[0.71-1.08] \\
n=50\end{array}$ \\
\hline $\begin{array}{l}\text { 2.2. Prothrombin time, } \\
\text { sec, ratio [6] }\end{array}$ & \multirow{2}{*}{ Thromborel S/Siemens } & $\begin{array}{c}1.06[0.93-1.18] \\
n=50\end{array}$ & $\begin{array}{c}1.04[0.95-1.15] \\
n=52\end{array}$ & $\begin{array}{c}1.01[0.94-1.14] \\
n=41\end{array}$ & $\begin{array}{c}1.02[0.95-1.12] \\
n=55\end{array}$ & $\begin{array}{c}1.01[0.92-1.14] \\
n=38\end{array}$ & $\begin{array}{c}0.98[0.90-1.05] \\
n=48\end{array}$ \\
\hline 2.3. PT Quick, \% [6] & & $\begin{array}{c}91[75-115] \\
n=50\end{array}$ & $\begin{array}{c}96[76-110] \\
n=50\end{array}$ & $99 \begin{array}{c}{[79-113]} \\
\mathrm{n}=41\end{array}$ & $\begin{array}{c}98[83-109] \\
n=55\end{array}$ & $\begin{array}{c}98[84-117] \\
n=37\end{array}$ & $\begin{array}{c}103[89-121] \\
n=48\end{array}$ \\
\hline $\begin{array}{l}\text { 2.4. Thrombin time, } \\
\text { sec, ratio [6] }\end{array}$ & $\begin{array}{c}\text { Test Thrombin Reagent/Sie- } \\
\text { mens }\end{array}$ & $\begin{array}{c}0.97[0.87-1.09] \\
n=50\end{array}$ & $\begin{array}{c}0.96[0.87-1.07] \\
n=52\end{array}$ & $\begin{array}{c}0.95[0.85-1.11] \\
n=41\end{array}$ & $\begin{array}{c}0.95[0.86-1.06] \\
n=55\end{array}$ & $\begin{array}{c}0.97[0.90-1.15] \\
n=38\end{array}$ & $\begin{array}{c}1.03[0.90-1.25] \\
n=51\end{array}$ \\
\hline $\begin{array}{l}\text { 2.5. Batroxobin time, } \\
\text { sec, ratio [6] }\end{array}$ & Batroxobin Reagent/Siemens & $\begin{array}{c}0.95[0.79-1.04] \\
n=40\end{array}$ & $\begin{array}{c}0.95[0.83-1.05] \\
n=46\end{array}$ & $\begin{array}{c}0.96[0.79-1.05] \\
n=35\end{array}$ & $\begin{array}{c}0.95[0.87-1.07] \\
n=56\end{array}$ & $\begin{array}{c}1.00[0.83-1.18] \\
n=35\end{array}$ & $\begin{array}{c}1.09[1.00-1.27] \\
n=45\end{array}$ \\
\hline \multicolumn{8}{|c|}{ 3. Blood-coagulation factors activity } \\
\hline 3.1. Factor II, \% [4] & $\begin{array}{l}\text { Coagulation Factor II Deficient } \\
\text { Plasma; Thromborel S/Siemens }\end{array}$ & $\begin{array}{c}108[86-144] \\
n=50\end{array}$ & $\begin{array}{c}117[88-150] \\
n=48\end{array}$ & $\begin{array}{c}140[100-200] \\
n=42\end{array}$ & $\begin{array}{c}135[105-178] \\
n=54\end{array}$ & $\begin{array}{c}139\left[\begin{array}{c}105-210] \\
n=36\end{array}\right. \\
\text { n }\end{array}$ & $\begin{array}{c}144[111-183] \\
n=48\end{array}$ \\
\hline 3.2. Factor $\mathrm{V}, \%[4]$ & $\begin{array}{l}\text { Coagulation Factor V Deficient } \\
\text { Plasma; Thromborel S/Siemens }\end{array}$ & $\begin{array}{c}86[50-140] \\
n=49\end{array}$ & $\begin{array}{c}70[40-120] \\
n=44\end{array}$ & $\begin{array}{c}68[26-150] \\
n=40\end{array}$ & $\begin{array}{c}46[14-82] \\
n=53\end{array}$ & $\begin{array}{c}58[25-122] \\
n=35\end{array}$ & $\begin{array}{c}80[37-150] \\
n=45\end{array}$ \\
\hline 3.3. Factor VII, \% [4] & $\begin{array}{l}\text { Coagulation Factor VII Deficient } \\
\text { Plasma; Thromborel S/Siemens }\end{array}$ & $\begin{array}{c}118[67-170] \\
n=53\end{array}$ & $\begin{array}{c}122[69-297] \\
n=48\end{array}$ & $\begin{array}{c}210[116-439] \\
n=42\end{array}$ & $\begin{array}{c}328[133-600] \\
n=54\end{array}$ & $\begin{array}{c}420\left[\begin{array}{c}153-600] \\
n=36\end{array}\right. \\
\end{array}$ & $\begin{array}{c}202[97-600] \\
n=48\end{array}$ \\
\hline 3.4. Factor VIII,\% [5] & $\begin{array}{l}\text { Coagulation Factor VIII Deficient } \\
\text { Plasma; Pathromtin SL/Siemens }\end{array}$ & $\begin{array}{c}84[49-144] \\
n=53\end{array}$ & $\begin{array}{c}105[50-155] \\
n=52\end{array}$ & $\begin{array}{c}126[78-222] \\
n=43\end{array}$ & $\begin{array}{c}167[90-256] \\
n=55\end{array}$ & $\begin{array}{c}180[94-344] \\
n=40\end{array}$ & $\begin{array}{c}175[113-390] \\
n=51\end{array}$ \\
\hline
\end{tabular}


Citation: Momot AP, Semenova NA, Belozerov DE, Trukhina DA, Kudinova IY (2016) The Dynamics of the Hemostatic Parameters in Physiological Pregnancy and After Delivery. J Hematol Blood Transfus Disord 3: 005

- Page 4 of $18 \cdot$

\begin{tabular}{|c|c|c|c|c|c|c|c|}
\hline 3.5. Factor $\mathrm{X}, \%[4]$ & $\begin{array}{l}\text { Coagulation Factor X Deficient } \\
\text { Plasma; Thromborel S/Siemens }\end{array}$ & $\begin{array}{c}93[74-132] \\
n=52\end{array}$ & $\begin{array}{c}100[75-160] \\
n=43\end{array}$ & $\begin{array}{c}125[92-168] \\
n=39\end{array}$ & $\begin{array}{c}155\left[\begin{array}{c}{[113-194]} \\
n=54\end{array}\right. \\
\text { n }\end{array}$ & $\begin{array}{c}168[119-244] \\
\mathrm{n}=33\end{array}$ & $\begin{array}{c}150[110-238] \\
\mathrm{n}=43\end{array}$ \\
\hline 3.6. Factor XI, \% [4] & $\begin{array}{l}\text { Coagulation Factor XI Deficient } \\
\text { Plasma; Pathromtin SL/Siemens }\end{array}$ & $\begin{array}{c}86[53-150] \\
n=49\end{array}$ & $\begin{array}{c}88[55-217] \\
n=50\end{array}$ & $\begin{array}{c}90[56-184] \\
\mathrm{n}=41\end{array}$ & $\begin{array}{c}80[49-172] \\
n=56\end{array}$ & $\begin{array}{c}85[50-207] \\
n=32\end{array}$ & $\begin{array}{c}92[61-180] \\
\mathrm{n}=44\end{array}$ \\
\hline 3.7. Factor XII, \% [4] & $\begin{array}{l}\text { Coagulation Factor XII Deficient } \\
\text { Plasma; Pathromtin SL/Siemens }\end{array}$ & $\begin{array}{c}82[47-143] \\
\mathrm{n}=48\end{array}$ & $\begin{array}{c}104[61-217] \\
n=45\end{array}$ & $\begin{array}{c}145[66-300] \\
n=36\end{array}$ & $\begin{array}{c}123[73-267] \\
n=54\end{array}$ & $\begin{array}{c}143[52-314] \\
n=32\end{array}$ & $\begin{array}{c}176[96-323] \\
n=40\end{array}$ \\
\hline 3.8. Factor XIII, \% [9] & Berichrom Factor XIII/Siemens & $\begin{array}{c}114[83-142] \\
n=47\end{array}$ & $\begin{array}{c}114[85-142] \\
n=49\end{array}$ & $\begin{array}{c}105[72-135] \\
n=37\end{array}$ & $\begin{array}{c}98[73-121] \\
n=51\end{array}$ & $\begin{array}{c}99[79-120] \\
n=37\end{array}$ & $\begin{array}{c}99[76-129] \\
n=41\end{array}$ \\
\hline \multicolumn{8}{|c|}{ 4. Fibrinogen concentration } \\
\hline 4.1. Fibrinogen, g/l [6] & Thromborel S/Siemens & $\begin{array}{c}3.21[2.50-4.41] \\
n=40\end{array}$ & $\begin{array}{c}4.41[3.25-5.47] \\
n=29\end{array}$ & $\begin{array}{c}4.93[3.64-6.28] \\
n=28\end{array}$ & $\begin{array}{c}5.22[4.21-6.13] \\
n=46\end{array}$ & $\begin{array}{c}6.15[4.65-7.37] \\
n=36\end{array}$ & $\begin{array}{c}5.94[4.56-7.90] \\
n=28\end{array}$ \\
\hline 4.2. Fibrinogen, $g / l[6]$ & Multifibren U/Siemens & $\begin{array}{c}2.92[2.08-4.05] \\
n=45\end{array}$ & $\begin{array}{c}4.10[2.38-5.21] \\
n=42\end{array}$ & $\begin{array}{c}4.75[2.80-6.28] \\
n=39\end{array}$ & $\begin{array}{c}5.13[3.70-6.48] \\
n=54\end{array}$ & $\begin{array}{c}6.36\left[\begin{array}{c}4.49-9.06] \\
n=36\end{array}\right.\end{array}$ & $\begin{array}{c}6.88[4.84-8.50] \\
n=45\end{array}$ \\
\hline \multicolumn{8}{|c|}{ 5. Activation markers of blood coagulation, thrombinemia, and fibrinolysis } \\
\hline $\begin{array}{l}\text { 5.1. Tissue factor, } \\
\text { activity, pmol// [10] }\end{array}$ & $\begin{array}{l}\text { Actichrome }{ }^{\circledast} \mathrm{TF} / \text { Sekisui Diag- } \\
\text { nostics (American Diagnostica) }\end{array}$ & $\begin{array}{c}2.9[1.5-11.1] \\
n=50\end{array}$ & $\begin{array}{c}2.9[1.4-9.8] \\
n=50\end{array}$ & $\begin{array}{c}3.6[1.6-13.6] \\
n=41\end{array}$ & $\begin{array}{c}6.7[2.2-32.5] \\
n=55\end{array}$ & $\begin{array}{c}7.9[2.8-100.8] \\
n=37\end{array}$ & $\begin{array}{c}8.9[4.0-213.8] \\
n=44\end{array}$ \\
\hline $\begin{array}{l}\text { 5.2. Factor VIla, activi- } \\
\text { ty, IU/ml [10] }\end{array}$ & $\begin{array}{l}\text { Assay Sense Human Factor VII } \\
\text { Chromo-genic Activity/Assay- } \\
\text { Pro, St. Charles, MO }\end{array}$ & $\begin{array}{c}0.56\left[\begin{array}{c}0.17-1.02] \\
n=48\end{array}\right. \\
\end{array}$ & $\begin{array}{c}0.65[0.19-2.68] \\
n=49\end{array}$ & $\begin{array}{c}0.79[0.16-3.44] \\
n=41\end{array}$ & $\begin{array}{c}0.97[0.17-3.00] \\
n=51\end{array}$ & $\begin{array}{c}0.87[0.31-3.06] \\
n=34\end{array}$ & $\begin{array}{c}1.20[0.59-2.35] \\
n=47\end{array}$ \\
\hline $\begin{array}{l}\text { 5.3. Fibrinopeptide A, } \\
\text { level, ng/ml [10] }\end{array}$ & $\begin{array}{l}\text { IMUCLONE FPA ELISA/Sekisui } \\
\text { Medical Co., Ltd }\end{array}$ & $\begin{array}{c}1.8[0.5-11.2] \\
n=45\end{array}$ & $\begin{array}{c}3.1[0.4-23.4] \\
n=48\end{array}$ & $\begin{array}{c}2.5[0.6-21.2] \\
n=39\end{array}$ & $\begin{array}{c}5.2[0.5-25.7] \\
n=55\end{array}$ & $\begin{array}{c}3.7[1.2-27.7] \\
n=33\end{array}$ & $\begin{array}{c}8.2[2.9-49.9] \\
n=44\end{array}$ \\
\hline $\begin{array}{l}\text { 5.4. D-Dimer, level, ng/ } \\
\mathrm{ml} \text { [3] }\end{array}$ & $\begin{array}{c}\text { Auto Blue D-dimer 400/Helena } \\
\text { Bioscience }\end{array}$ & $\begin{array}{c}109[32-227] \\
n=50\end{array}$ & $\begin{array}{c}166[32-311] \\
n=45\end{array}$ & $\begin{array}{c}171[62-348] \\
n=37\end{array}$ & $\begin{array}{c}277[69-488] \\
n=50\end{array}$ & $\begin{array}{c}374[122-699] \\
\mathrm{n}=40\end{array}$ & $\begin{array}{c}538\left[\begin{array}{c}200-1120] \\
\mathrm{n}=44\end{array}\right. \\
\text { (1) }\end{array}$ \\
\hline $\begin{array}{l}\text { 5.5. D-Dimer, level, ng/ } \\
\mathrm{ml} \text { [6] }\end{array}$ & $\begin{array}{l}\text { Auto Red D-dimer 70/Helena } \\
\text { Bioscience }\end{array}$ & $\begin{array}{c}71[10-181] \\
n=48\end{array}$ & $\begin{array}{c}84[10-253] \\
n=46\end{array}$ & $\begin{array}{c}131[30-311] \\
n=41\end{array}$ & $\begin{array}{c}271[152-561] \\
n=53\end{array}$ & $\begin{array}{c}330[135-771] \\
\mathrm{n}=37\end{array}$ & $\begin{array}{c}385[206-959] \\
n=40\end{array}$ \\
\hline $\begin{array}{l}\text { 5.6. D-Dimer, level, ng/ } \\
\mathrm{ml} \text { [11] }\end{array}$ & D-dimer Nycocard/Axis Shield & $\begin{array}{c}100[100-300] \\
n=54\end{array}$ & $\begin{array}{c}100[100-300] \\
n=54\end{array}$ & $\begin{array}{c}100[100-200] \\
n=42\end{array}$ & $\begin{array}{c}100[100-460] \\
n=57\end{array}$ & $\begin{array}{c}200[100-430] \\
n=37\end{array}$ & $\begin{array}{c}200[100-865] \\
n=48\end{array}$ \\
\hline $\begin{array}{l}\text { 5.7. D-Dimer, level, } \\
\text { ngFEU/ml [8] }\end{array}$ & $\begin{array}{l}\text { IMMULITE } 2000 \text { D-Dimer/ } \\
\text { Siemens }\end{array}$ & $\begin{array}{c}191[50-837] \\
n=52\end{array}$ & $\begin{array}{c}223[85-816] \\
n=47\end{array}$ & $\begin{array}{c}255[112-1000] \\
n=30\end{array}$ & $\begin{array}{c}640[357-1458] \\
n=27\end{array}$ & $\begin{array}{c}975[420-2290] \\
n=20\end{array}$ & Not reported \\
\hline \multicolumn{8}{|c|}{ 6. Physiological anticoagulants } \\
\hline $\begin{array}{l}\text { 6.1. Antithrombin III, } \\
\text { activity, \% [6] }\end{array}$ & $\begin{array}{c}\text { Berichrom Antithrombin III (A)/ } \\
\text { Siemens }\end{array}$ & $\begin{array}{c}109[89-119] \\
n=48\end{array}$ & $\begin{array}{c}103[92-119] \\
n=50\end{array}$ & $\begin{array}{c}102[89-118] \\
n=38\end{array}$ & $\begin{array}{c}105[90-119] \\
n=55\end{array}$ & $\begin{array}{c}107[89-124] \\
n=37\end{array}$ & $\begin{array}{c}119[91-138] \\
n=48\end{array}$ \\
\hline $\begin{array}{l}\text { 6.2. Screening for } \\
\text { abnormalities of protein } \\
\text { C system, NR [6] }\end{array}$ & ProC Global/Siemens & $\begin{array}{c}1.05[0.80-1.39] \\
\mathrm{n}=46\end{array}$ & $\begin{array}{c}1.08[0.78-1.36] \\
n=50\end{array}$ & $\begin{array}{c}0.99[0.74-1.44] \\
n=40\end{array}$ & $\begin{array}{c}0.90[0.74-1.05] \\
n=55\end{array}$ & $\begin{array}{c}0.82\left[\begin{array}{l}0.65-1.00] \\
\mathrm{n}=40\end{array}\right. \\
\text { n }\end{array}$ & $\begin{array}{c}0.80\left[\begin{array}{l}0.57-1.12] \\
n=46\end{array}\right. \\
\end{array}$ \\
\hline $\begin{array}{l}\text { 6.3. Protein C, activity, } \\
\%[6]\end{array}$ & Berichrom Protein C/Siemens & $\begin{array}{c}104[80-132] \\
n=48\end{array}$ & $\begin{array}{c}109[85-136] \\
n=50\end{array}$ & $\begin{array}{c}119[99-146] \\
n=40\end{array}$ & $\begin{array}{c}122[97-152] \\
n=55\end{array}$ & $\begin{array}{c}118[99-160] \\
n=33\end{array}$ & $\begin{array}{c}142\left[\begin{array}{c}{[112-183]} \\
n=45\end{array}\right. \\
\text { n }\end{array}$ \\
\hline $\begin{array}{l}\text { 6.4. Protein C, activity, } \\
\text { \% [6] }\end{array}$ & $\begin{array}{l}\text { Protein C Reagent COAG/ } \\
\text { Siemens }\end{array}$ & $\begin{array}{c}92[73-120] \\
n=50\end{array}$ & $\begin{array}{c}91[72-125] \\
n=50\end{array}$ & $\begin{array}{c}122[97-158] \\
n=42\end{array}$ & $\begin{array}{c}131[79-161] \\
n=55\end{array}$ & $\begin{array}{c}102[79-151] \\
n=38\end{array}$ & $\begin{array}{c}144[100-214] \\
n=49\end{array}$ \\
\hline $\begin{array}{l}\text { 6.5. Protein S Free, } \\
\text { activity, \% [10] }\end{array}$ & $\begin{array}{l}\text { IMUCLONE Free Protein S } \\
\text { ELISA/Sekisui Diagnostics } \\
\text { (American Diagnostica) }\end{array}$ & $\begin{array}{c}117[64-143] \\
n=48\end{array}$ & $\begin{array}{l}67.7[47-122] \\
n=50\end{array}$ & $\begin{array}{c}72[56-121] \\
n=41\end{array}$ & $\begin{array}{c}64[39-108] \\
n=54\end{array}$ & $\begin{array}{c}65[37-102] \\
n=38\end{array}$ & $\begin{array}{c}61[45-114] \\
n=43\end{array}$ \\
\hline $\begin{array}{l}\text { 6.6. Thrombomodulin } \\
\text { (soluble receptor } \\
\text { CD141), level, ng/ } \\
\text { ml [10] }\end{array}$ & $\begin{array}{l}\text { CD141 ELISA KIT/BCM Diag- } \\
\text { nostics }\end{array}$ & $\begin{array}{c}3.25[2.19-6.57] \\
n=48\end{array}$ & $\begin{array}{c}3.40[2.38-6.74] \\
n=48\end{array}$ & $\begin{array}{c}3.32[1.77-8.02] \\
n=40\end{array}$ & $\begin{array}{c}3.05[2.26-7.41] \\
n=52\end{array}$ & $\begin{array}{c}3.52[2.48-8.05] \\
n=37\end{array}$ & $\begin{array}{c}3.50[1.67-7.30] \\
n=45\end{array}$ \\
\hline $\begin{array}{c}\text { 6.7. Tissue factor path- } \\
\text { way inhibitor, activity, } \\
\text { U/ml [10] }\end{array}$ & $\begin{array}{c}\text { ACTICHROME }{ }^{\oplus} \text { TFPI/Sekisui } \\
\text { Diagnostics (American Diag- } \\
\text { nostica) }\end{array}$ & $\begin{array}{c}1.58[1.07-2.99] \\
n=48\end{array}$ & $\begin{array}{c}1.50[0.75-3.33] \\
n=50\end{array}$ & $\begin{array}{c}1.68[0.85-3,69] \\
n=43\end{array}$ & $\begin{array}{c}1.66[0.95-3.84] \\
n=55\end{array}$ & $\begin{array}{c}1.96\left[\begin{array}{c}1.13-3.06] \\
n=35\end{array}\right. \\
\end{array}$ & $\begin{array}{c}2.72[1.54-5.18] \\
n=45\end{array}$ \\
\hline \multicolumn{8}{|c|}{ 7. Assessment methods of fibrinolytic reactions } \\
\hline $\begin{array}{l}\text { 7.1. Plasminogen, } \\
\text { level, \% [6] }\end{array}$ & $\begin{array}{l}\text { Berichrom Plasminogen/Sie- } \\
\text { mens }\end{array}$ & $\begin{array}{c}106[84-135] \\
n=48\end{array}$ & $\begin{array}{c}116[85-140] \\
n=51\end{array}$ & $\begin{array}{c}138[110-171] \\
n=38\end{array}$ & $\begin{array}{c}147\left[\begin{array}{l}109-186] \\
n=53\end{array}\right. \\
\end{array}$ & $\begin{array}{c}161[111-199] \\
\mathrm{n}=37\end{array}$ & $\begin{array}{c}155\left[\begin{array}{c}{[118-189]} \\
\mathrm{n}=48\end{array}\right. \\
\end{array}$ \\
\hline $\begin{array}{l}\text { 7.2. Tissue-type } \\
\text { plasminogen activator, } \\
\text { level, } \mathrm{ng} / \mathrm{ml}[10]\end{array}$ & $\begin{array}{l}\text { t-PA Combi Actibind ELISA/ } \\
\text { Technoclone GmbH }\end{array}$ & $\begin{array}{c}5.2[1.1-14.9] \\
n=48\end{array}$ & $\begin{array}{c}6.0[1.0-15.0] \\
n=49\end{array}$ & $\begin{array}{c}7.3[0.9-20.3] \\
\mathrm{n}=41\end{array}$ & $\begin{array}{c}8.3[2.2-25.1] \\
n=54\end{array}$ & $\begin{array}{c}10.3[1.9-26.3] \\
n=37\end{array}$ & $\begin{array}{c}7.6[1.8-19.1] \\
n=43\end{array}$ \\
\hline $\begin{array}{c}\text { 7.3. Plasminogen acti- } \\
\text { vator inhibitor } 1 \text {, level, } \\
U / m l[10]\end{array}$ & $\begin{array}{l}\text { TECHNOZYM } \text { PAl-1 Actibind }^{\circledast} \\
\text { ELISA/Technoclone GmbH }\end{array}$ & $\begin{array}{c}3.0[2.3-11.0] \\
n=48\end{array}$ & $\begin{array}{c}2.8[2.3-7.2] \\
\mathrm{n}=50\end{array}$ & $\begin{array}{c}3.0[2.5-8.6] \\
n=42\end{array}$ & $\begin{array}{l}4.5[2.8-14.1] \\
n=57\end{array}$ & $\begin{array}{c}15.3[3.0-54.9] \\
n=37\end{array}$ & $\underset{n=46}{2.7}$ \\
\hline $\begin{array}{l}\text { 7.4. Urokinase-type } \\
\text { plasminogen activator, } \\
\text { level, } \mathrm{ng} / \mathrm{ml}[10]\end{array}$ & \multirow{2}{*}{$\begin{array}{l}\text { u-PA Combi Actibind ELISA/ } \\
\text { Technoclone GmbH }\end{array}$} & $\begin{array}{c}1.29[0.41-2.80] \\
n=51\end{array}$ & $\begin{array}{c}1.32[0.77-2.19] \\
n=51\end{array}$ & $\begin{array}{c}1.39[0.71-2.00] \\
n=41\end{array}$ & $\begin{array}{c}1.45[0.82-2.95] \\
n=55\end{array}$ & $\begin{array}{c}1.92[1.51-5.88] \\
n=38\end{array}$ & $\begin{array}{c}1.10[0.79-1.54] \\
n=43\end{array}$ \\
\hline $\begin{array}{l}\text { 7.5. Urokinase-type } \\
\text { plasminogen activator, } \\
\text { activity, } \mathrm{U} / \mathrm{ml}[10]\end{array}$ & & $\begin{array}{c}0.18[0.07-0.38] \\
n=51\end{array}$ & $\begin{array}{c}0.16[0.06-0.28] \\
n=51\end{array}$ & $\begin{array}{c}0.13[0.06-0.25] \\
n=42\end{array}$ & $\begin{array}{c}0.15[0.08-0.35] \\
n=55\end{array}$ & $\begin{array}{c}0.28[0.15-0.52] \\
n=38\end{array}$ & $\begin{array}{c}0.12[0.06-0.18] \\
n=46\end{array}$ \\
\hline $\begin{array}{l}\text { 7.6. Thrombin activated } \\
\text { fibrinolysis inhibitor, } \\
\text { level, \% [10] }\end{array}$ & $\begin{array}{c}\text { IMUCLONE TAFI ELISA/ } \\
\text { Sekisui Diagnostics (American } \\
\text { Diagnostica) }\end{array}$ & $\begin{array}{c}107[49-153] \\
\quad \mathrm{n}=50\end{array}$ & $\begin{array}{c}113[63-163] \\
n=50\end{array}$ & $\begin{array}{c}127[78-196] \\
\mathrm{n}=42\end{array}$ & $\begin{array}{c}138\left[\begin{array}{c}103-200] \\
n=55\end{array}\right. \\
\text { na }\end{array}$ & $\begin{array}{c}158[106-245] \\
n=39\end{array}$ & $\begin{array}{c}148[80-199] \\
n=45\end{array}$ \\
\hline
\end{tabular}


Citation: Momot AP, Semenova NA, Belozerov DE, Trukhina DA, Kudinova IY (2016) The Dynamics of the Hemostatic Parameters in Physiological Pregnancy and After Delivery. J Hematol Blood Transfus Disord 3: 005

- Page 5 of $18 \bullet$

\begin{tabular}{|c|c|c|c|c|c|c|c|}
\hline $\begin{array}{l}\text { 7.7. } \alpha 2 \text {-Antiplasmin, } \\
\text { activity, \% [6] }\end{array}$ & $\begin{array}{l}\text { Berichrom a2-Antiplasmin/ } \\
\text { Siemens }\end{array}$ & $\begin{array}{c}113[80-131] \\
n=45\end{array}$ & $\begin{array}{c}113[79-132] \\
n=34\end{array}$ & $\begin{array}{c}93[62-124] \\
n=37\end{array}$ & $\begin{array}{c}67[45-93] \\
n=53\end{array}$ & $\begin{array}{c}62[46-94] \\
n=34\end{array}$ & $\begin{array}{c}65[50-129] \\
n=37\end{array}$ \\
\hline \multicolumn{8}{|c|}{ 8. Thrombin generation test (calibrated throbogram) [7] } \\
\hline $\begin{array}{l}\text { 8.2. Time to reach peak } \\
\text { thrombin (tt-Peak), min }\end{array}$ & \multirow{3}{*}{$\begin{array}{c}\text { PPP-Reagent } 5 \mathrm{pM} \text {, Thrombin } \\
\text { Calibrator, FluCa-Kit/Thrombi- } \\
\text { noscope BV }\end{array}$} & $\begin{array}{c}5.96[4.50-9.47] \\
n=48\end{array}$ & $\begin{array}{c}5.16\left[\begin{array}{c}4.06-7.79] \\
n=48\end{array}\right. \\
\text { a }\end{array}$ & $\begin{array}{c}5.72[4.17-7.34] \\
n=40\end{array}$ & $\begin{array}{c}5.16\left[\begin{array}{c}{[4.00-6.33]} \\
n=54\end{array}\right. \\
\end{array}$ & $\begin{array}{c}5.25[3.67-7.51] \\
n=38\end{array}$ & $\begin{array}{c}4.49[3.65-6.00] \\
n=45\end{array}$ \\
\hline $\begin{array}{l}\text { 8.3. Peak thrombin, } \\
\mathrm{nmol} / \mathrm{l}\end{array}$ & & $\begin{array}{c}186[51-341] \\
n=48\end{array}$ & $\begin{array}{c}290[147-368] \\
n=48\end{array}$ & $\begin{array}{c}283[227-445] \\
n=40\end{array}$ & $\begin{array}{c}325[224-480] \\
n=54\end{array}$ & $\begin{array}{c}318[204-488] \\
n=38\end{array}$ & $\begin{array}{c}376[271-485] \\
n=45\end{array}$ \\
\hline $\begin{array}{l}\text { 8.4. Endogenous } \\
\text { Thrombin Potential } \\
\text { (ETP), nmol ×min }\end{array}$ & & $\begin{array}{c}1105[589-1770] \\
n=48\end{array}$ & $\begin{array}{c}1542[895-2165] \\
n=48\end{array}$ & $\begin{array}{c}1605[1015-2444] \\
n=40\end{array}$ & $\begin{array}{c}1723[1158-3072] \\
n=54\end{array}$ & $\begin{array}{c}1744[993-2850] \\
n=38\end{array}$ & $\begin{array}{c}1621[1165-2245] \\
n=45\end{array}$ \\
\hline
\end{tabular}

Table 1: Reference range of different methods to study hemostatic system.

Notes: $[\mathrm{x}$ ] - Data is presented as a median; in square brackets there's $2.5 \%-97.5 \%$ interval; FEU - Fibrinogen Equivalent Units; $n$ - number of observations; used equipment is presented in the superior position; ADP - Adenosine Diphosphate; APTT - Activated Partial Thromboplastin Time; IU - International Unit; ratio is calculated in pregnant women enrolled in the study as a quotient of a parameter of coagulation time to a parameter in the sample of normal control plasma; NR - Normalized Ratio; ADAMTS-13 - metalloproteinase which cleaves von Willebrand factor ("a disintegrin and metalloproteinase with a thrombospondin type 1 motif, member 13"); VIla - activated factor VII.

Besides, Standard Human Plasma (Siemens) was used to determine the parameters in the table under № 1.4.3, 3.1-3.8, 6.1-6.4, 7.1, 7.7 .

\section{Statistical calculation}

The statistical analysis of data was done by using the programs SPSS 16.0 for Windows (SPSS Inc., USA) and STATISTICA 6.1 (Statsoft Inc., Tulsa, USA). To test normally distributed data Kolmogorov-Smirnov and Shapiro-Wilk tests were used. The data from different laboratory methods were found to be of abnormal distribution. Nonparametric tests (Kruskal Wallis and Mann-Whitney $\mathrm{U}$ tests) were used for comparative analysis. Reference intervals $\left(2.5^{\text {th }}\right.$ and $97.5^{\text {th }}$ percentiles with confidential interval 95\%) were calculated for each test in each time point. Spearman rank correlation coefficients were used. The differences were considered statistically significant when the $\mathrm{p}$ value obtained was $<0.05$.

\section{Results}

The values in the table 1 describe a large number of hemostatic parameters at different stages of pregnancy and after spontaneous labor. For comparison, values are also given for healthy, nonpregnant women.

According to the table 1, platelet count in blood, Level 4 of a Plate Factor (PF 4), as well as the content of endothelin 1 remain stable and are not influenced by the onset of pregnancy and its course.

The same applies to the results of determination in screening coagulation tests (Activated Partial Thromboplastin Time (APTT), prothrombin time, thrombin time, batroxobin time), in the assessment of Factor XI activity, as well as antithrombin III activity. The level of free thrombomodulin (soluble receptor CD141) in blood plasma and tissue-type Plasminogen Activator (t-PA) was also stable. The study presents only the parameters, which were changed during pregnancy with negative, positive or multidirectional dynamics confirmed by statistically significant differences of the results at different phases of the study (Figures 1-5). In some cases figures are given to show some parameters without significant changes during pregnancy (platelet aggregation with ADP in a dose of $2.0 \mu \mathrm{mol} / \mathrm{l}$, antithrombin III, thrombomodulin, and tissue-type plasminogen activator), it was interesting to make comparative analysis.

In figures 1-5, box plots represent the range of data from the $25^{\text {th }}$ to $75^{\text {th }}$ percentiles, while the bar in the middle of each box plot represents the median value obtained excluding outliers. Circles indicate outliers (1.5xthe interquartile range) and extreme values (3.0xthe interquartile range) outside the central box, respectively.

\section{Parameters of primary hemostasis and homocysteine}

Platelet aggregation in platelet enriched plasma used as an agonist of low dose $(0.1 \mu \mathrm{mol})$ disodium salt of Adenosine Diphosphate (ADP) increased in women during pregnancy compared to women in the pregravid period (Figure 1a). This increase was observed from early pregnancy (12-13 weeks, by 1.9 times) and reached its maximum in late pregnancy. At the same time, if the dose of ADP was changed into $2.0 \mu \mathrm{mol}$ or other reagents (adrenalin, collagen or ristocetin) were used to stimulate platelet aggregation, the differences in the results between pregnant and nonpregnant women were not observed (Figure $1 \mathrm{~b}$ and table 1 ). It should be noted that the level of PF4 in blood plasma among pregnant women was the same as in the pregravid period. These peculiarities of platelet aggregation were accompanied by the gradual increase of von Willebrand factor antigen (vWF Ag) level that was measured by two different kits of reagents (Figures $1 \mathrm{c}$ and d). The obtained results were correlated with each other (correlation coefficient $0.34 ; \mathrm{p}<0.001$ ) but they had different reference intervals at different stages of pregnancy. Ristocetin Cofactor Activity von Willebrand Factor (RCoA) also grew up from 12-13 weeks of pregnancy and increased compare to baseline values (pregravid period) by 1.18 times at $22-24$ weeks and by 1.41 times at 34-36 weeks (Figure 1e). Correlation coefficient between platelet aggregation activity (ADP in dose $0.1 \mu \mathrm{mol}$ ) and RCoA or vWF Ag was correspondingly $0.17(\mathrm{p}=0.025)$ and $0.34(\mathrm{p}<0.001)$.

The obtained results also indicated the stable level and activity of metalloproteinase ADAMTS-13 [metalloproteinase which cleaves von Willebrand factor "a disintegrin and metalloproteinase with a thrombospondin type motif, member 13"] at 6-8 weeks and 12-13 weeks, which were not different from the results of women in pregravid period (Figures $1 \mathrm{f}$ and $\mathrm{g}$ ). Then, starting from the $22 \mathrm{~d}$ week to the late pregnancy, noticeable reduction of this metalloproteinase was observed by $28.7-31.7 \%$ (antigen) and by $26.4-39.2 \%$ (activity) compared to the data in pregravid period.

In the performed studies correlation coefficient between RCoA and ADAMTS-13 activity was $0.27(\mathrm{p}<0.001)$ and between antigens vWF and metalloproteinase ADAMTS-13 (with reagent vWF AG) was $0.32(\mathrm{p}<0.001)$.

During pregnancy homocysteine concentration was decreasing since early pregnancy (6-8 weeks) by $31.1 \%$ compared to its amount in pregravid period (Figure $1 \mathrm{~h}$ ). At a later stage this decrease continued and the lowest concentration of homocysteine was identified in the period from 22 to 36 weeks of pregnancy. It should be noted that all 
Citation: Momot AP, Semenova NA, Belozerov DE, Trukhina DA, Kudinova IY (2016) The Dynamics of the Hemostatic Parameters in Physiological Pregnancy and After Delivery. J Hematol Blood Transfus Disord 3: 005

a)

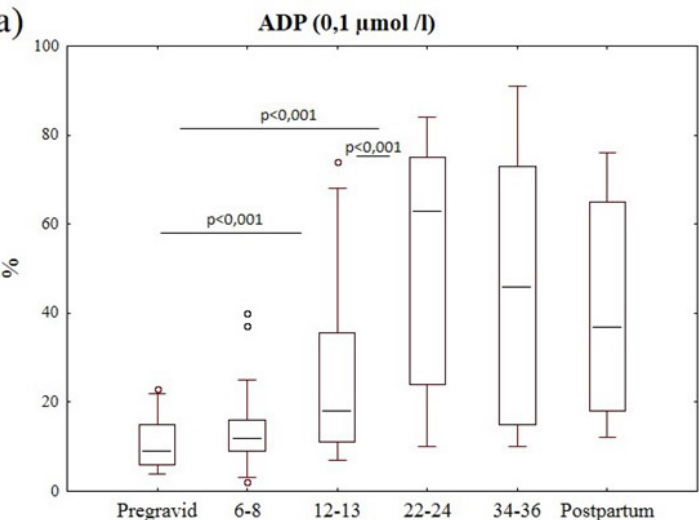

c)

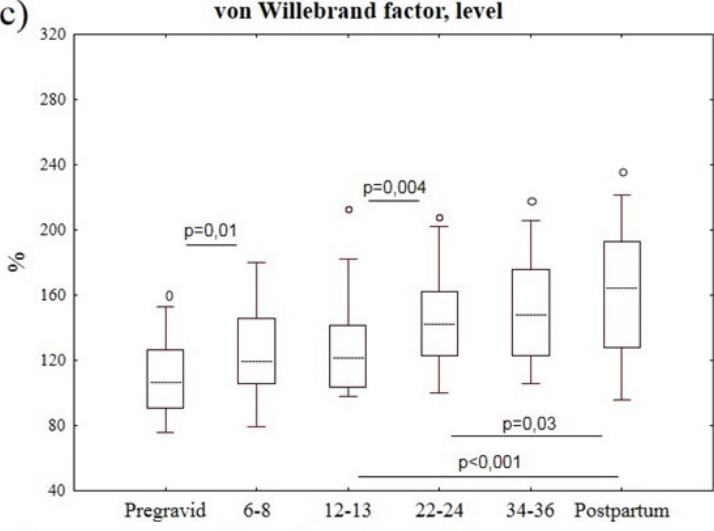

e)

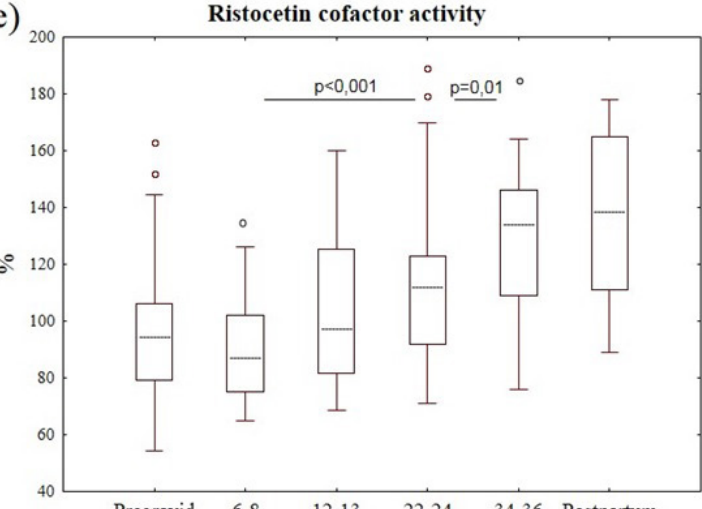

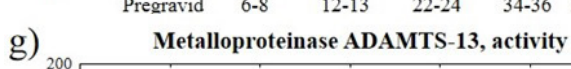

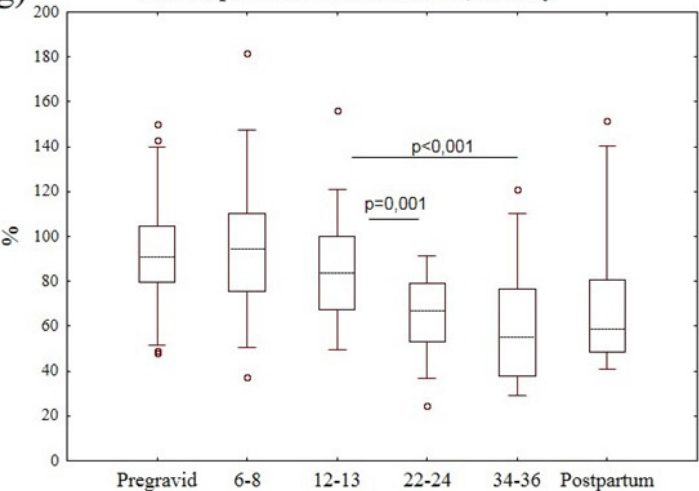

b)

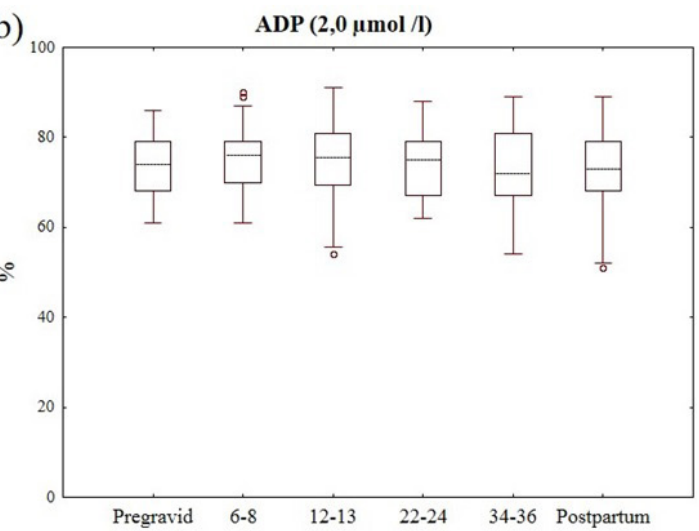

d)
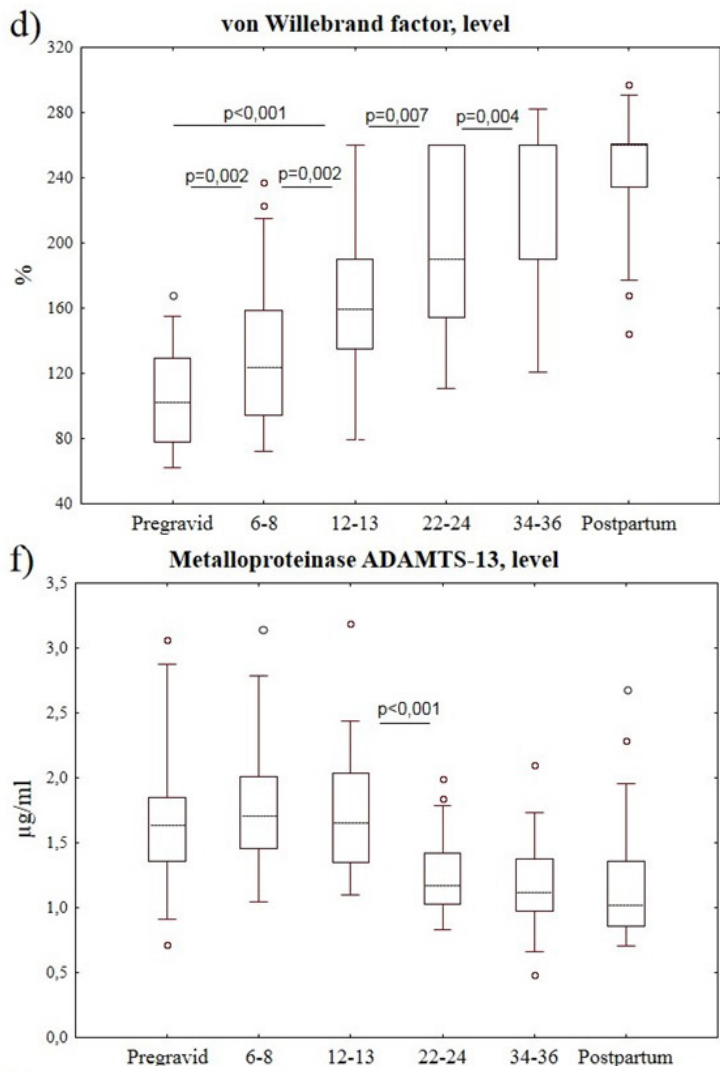

h)

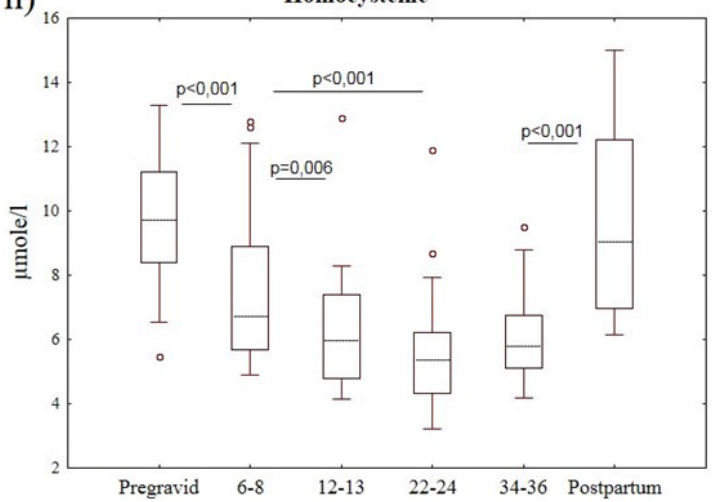

Figure 1: Box plots of reference intervals in pregravid period, different stages of pregnancy and after delivery for, a) ADP (0.1 $\mu \mathrm{mol} / \mathrm{l})$; b) $A D P(2.0 \mu \mathrm{mol} / \mathrm{I})$; c) von Willebrand factor level (reagent-«TECHNOZYM vWF: Ag ELISA»); d) von Willebrand factor level (reagent-«vWF Ag»); e) ristocetin cofactor activity; f) metalloproteinase ADAMTS-13, level; g) metalloproteinase ADAMTS-13, activity; h) homocysteine. 
Citation: Momot AP, Semenova NA, Belozerov DE, Trukhina DA, Kudinova IY (2016) The Dynamics of the Hemostatic Parameters in Physiological Pregnancy and After Delivery. J Hematol Blood Transfus Disord 3: 005

a)

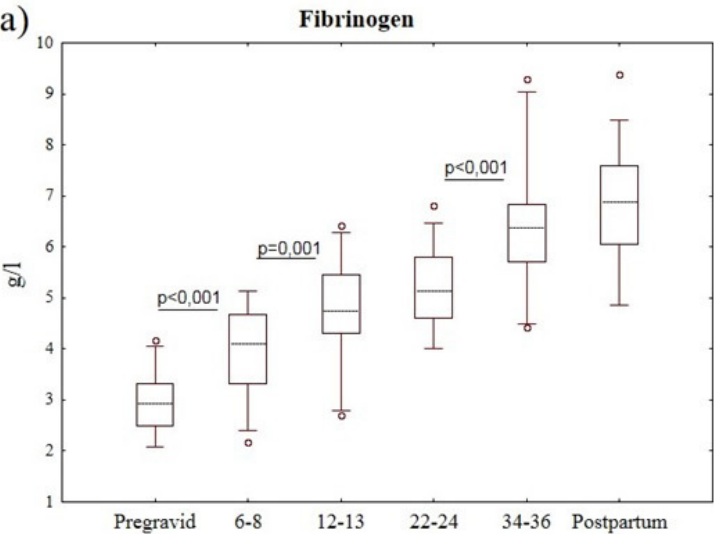

c)

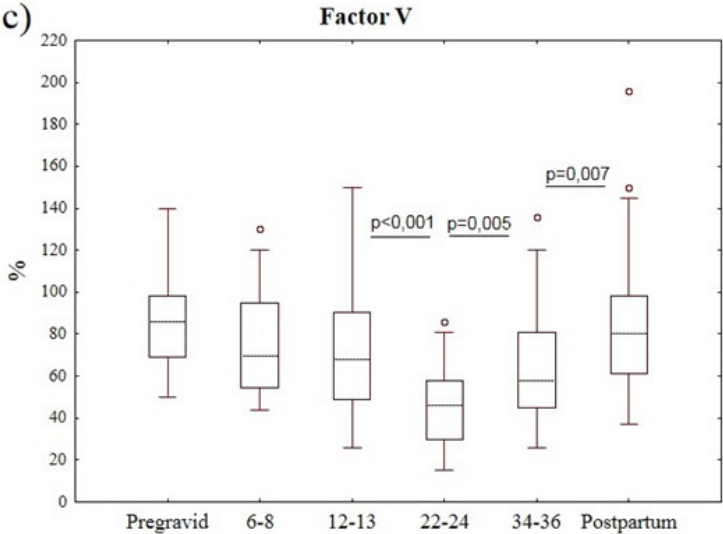

e)

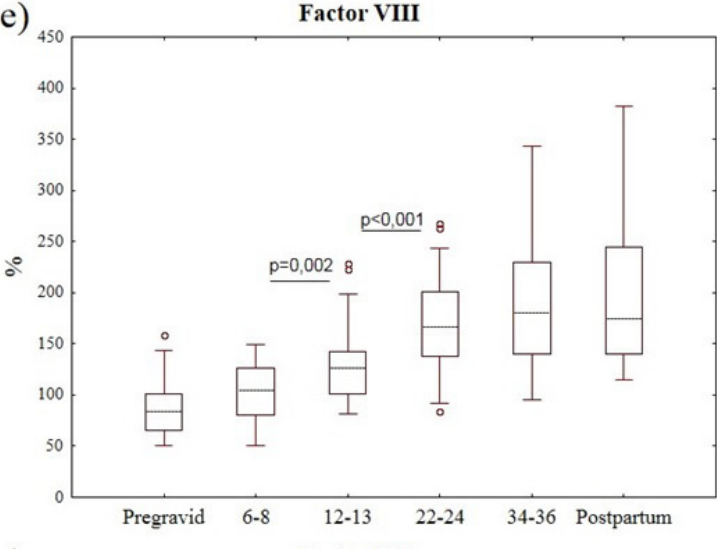

g)

Factor XII

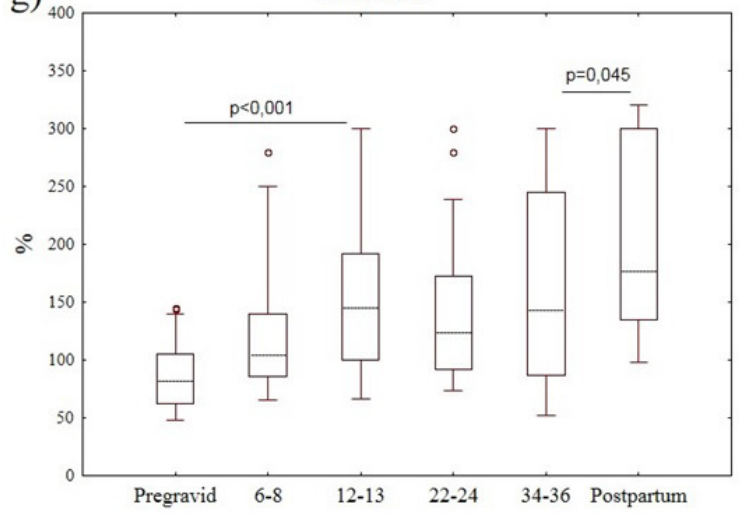

b)

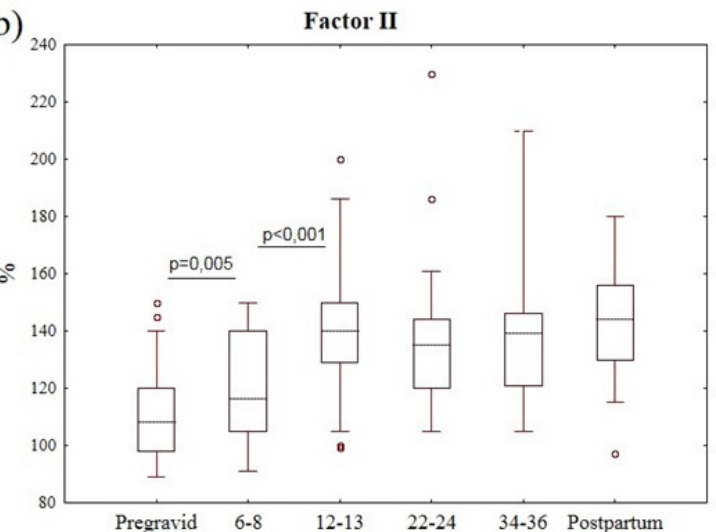

d)

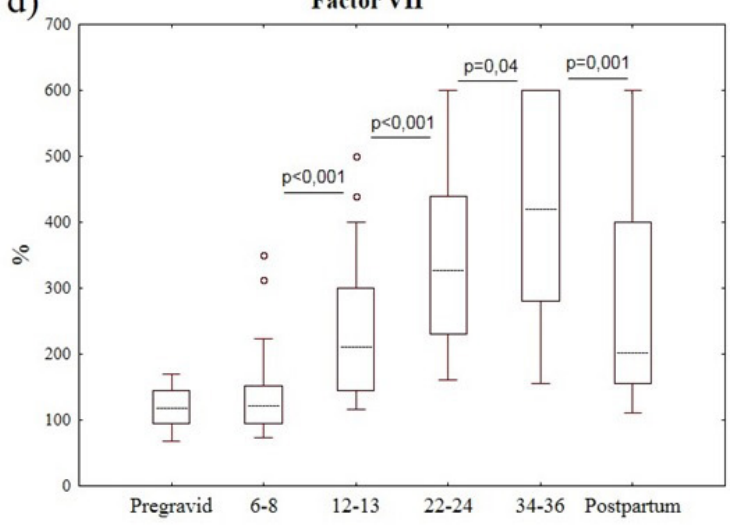

f)
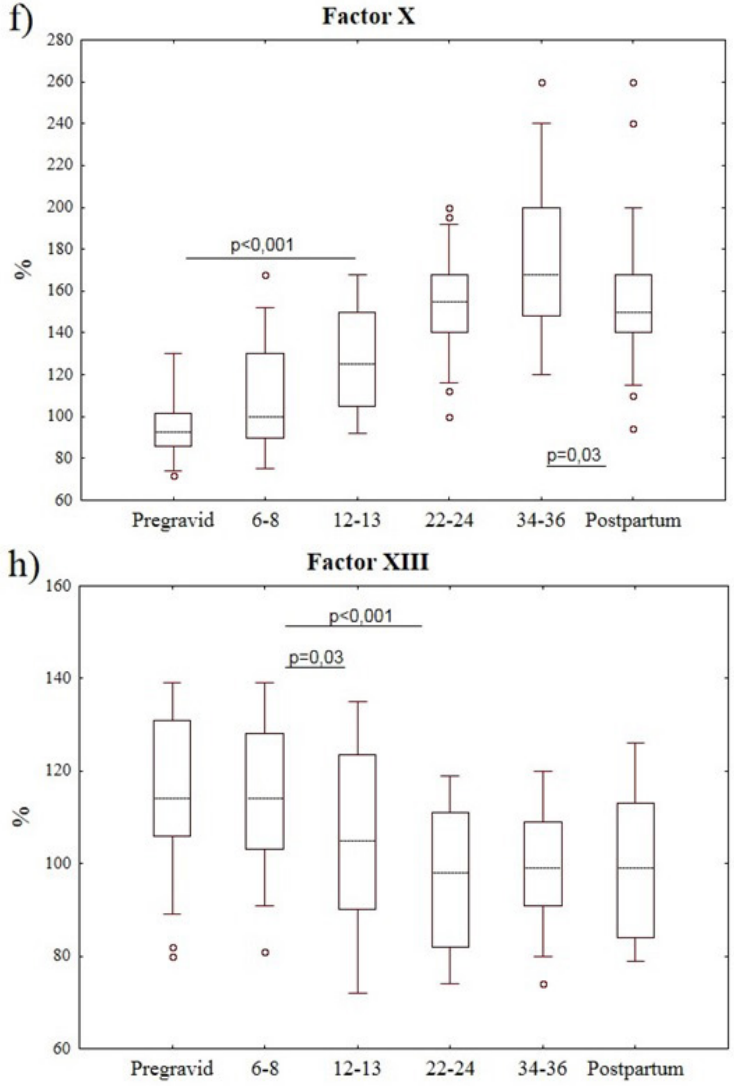

Figure 2: Box plots of reference intervals in pregravid period, different stages of pregnancy and after delivery for a) fibrinogen (reagent-«Multifibren $U ») ; b$ ) factor II; c) factor V; d) factor VII; e) factor VIII; f) factor X; g) factor XII; h) factor XIII. 
Citation: Momot AP, Semenova NA, Belozerov DE, Trukhina DA, Kudinova IY (2016) The Dynamics of the Hemostatic Parameters in Physiological Pregnancy and After Delivery. J Hematol Blood Transfus Disord 3: 005

a)

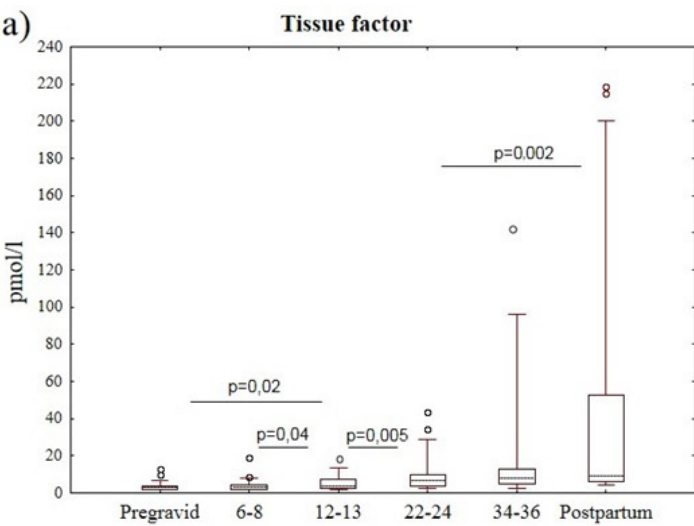

c)

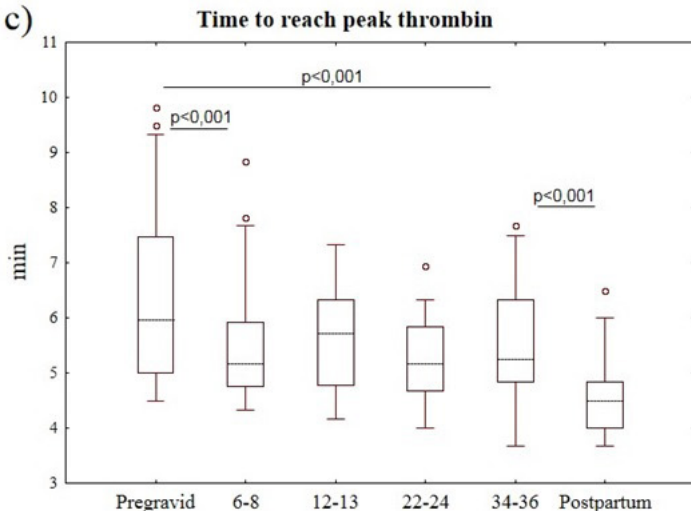

e)

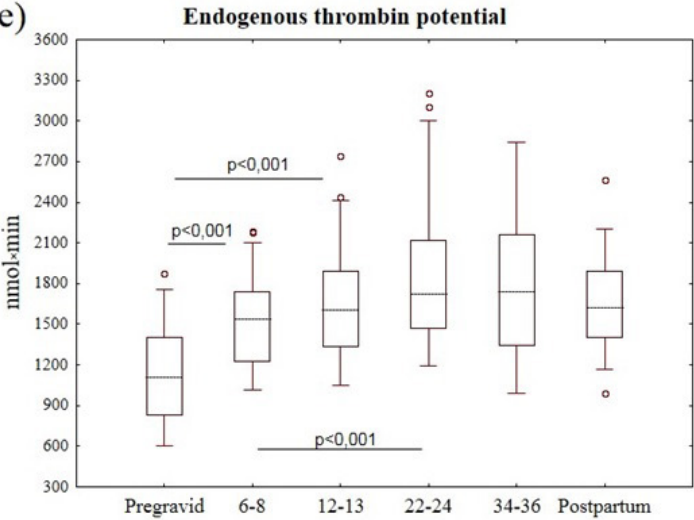

g)

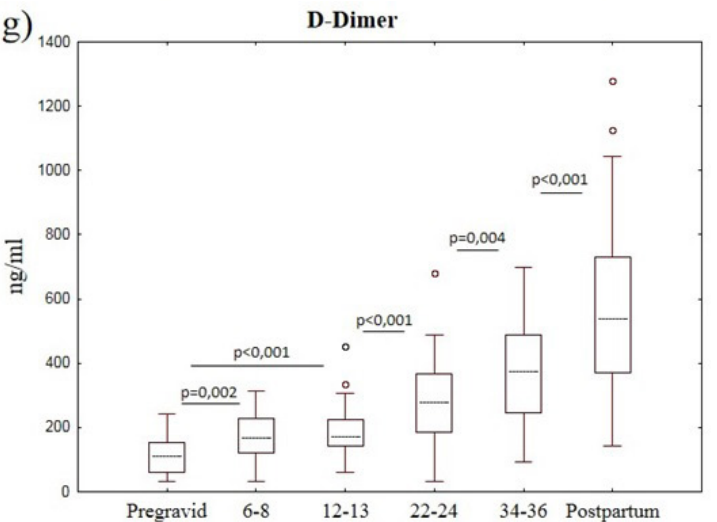

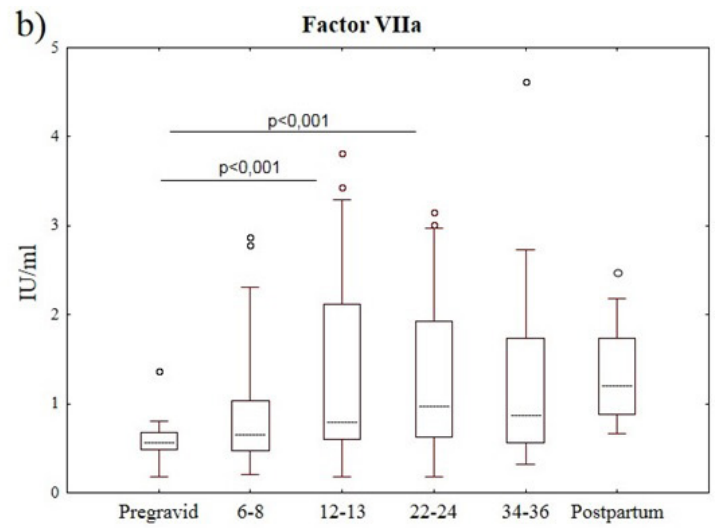
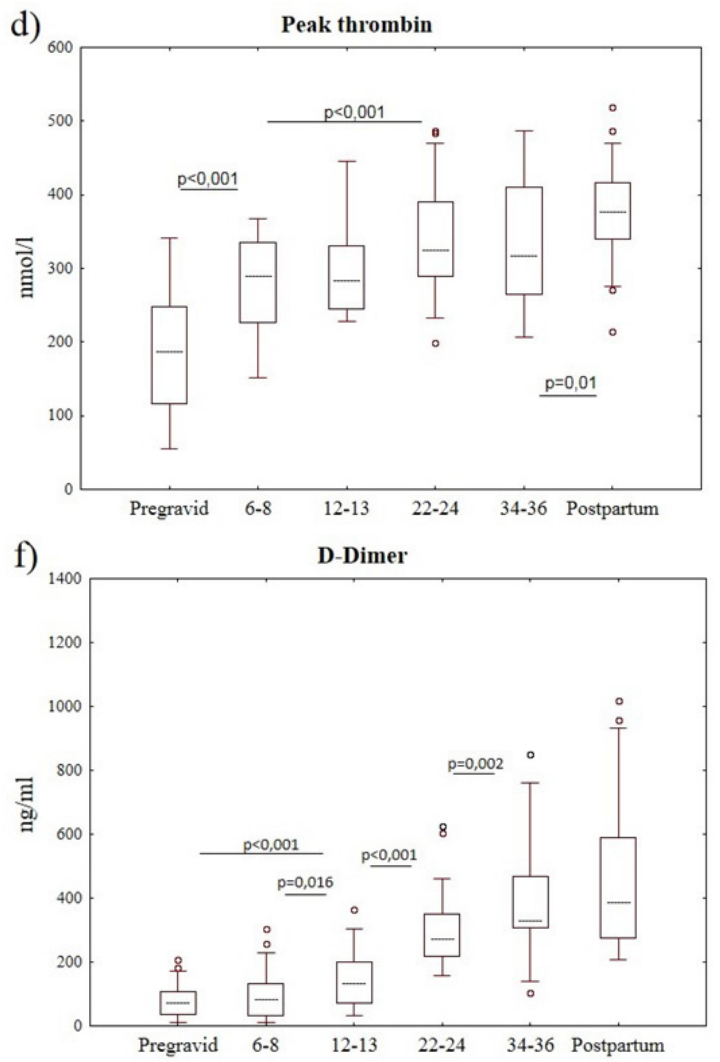

h)

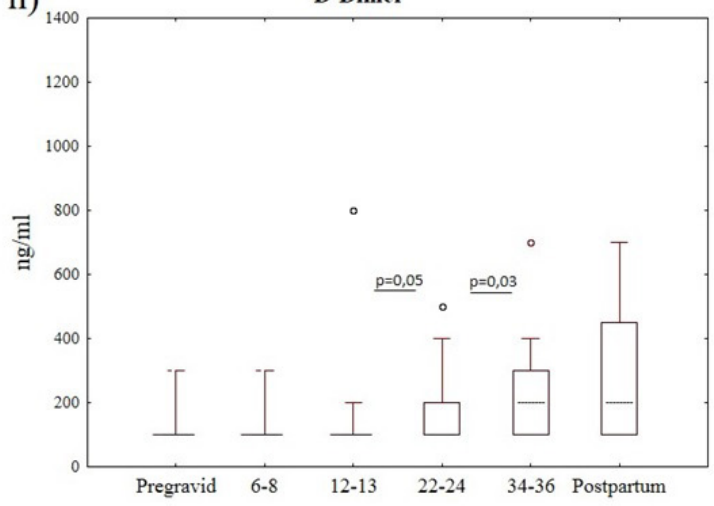

Figure 3: Box plots of reference intervals in pregravid period, different stages of pregnancy and after delivery for a) tissue factor; b) factor VIla; c) time to reach peak thrombin; d) peak thrombin; e) endogenous thrombin potential; f) D-Dimer (reagent-«Auto Red D-dimer 700»); g) D-Dimer (reagent-«Auto Blue D-Dimer 400»); h) D-Dimer (reagent-«D-dimer Nycocard»). 
Citation: Momot AP, Semenova NA, Belozerov DE, Trukhina DA, Kudinova IY (2016) The Dynamics of the Hemostatic Parameters in Physiological Pregnancy and After Delivery. J Hematol Blood Transfus Disord 3: 005

a)

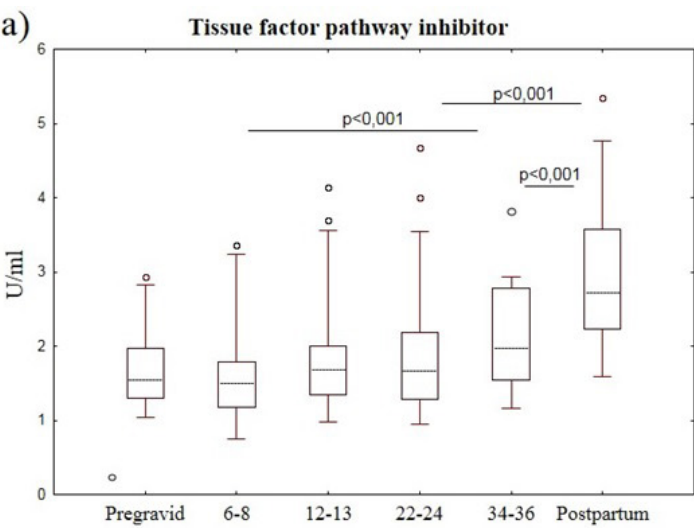

c)

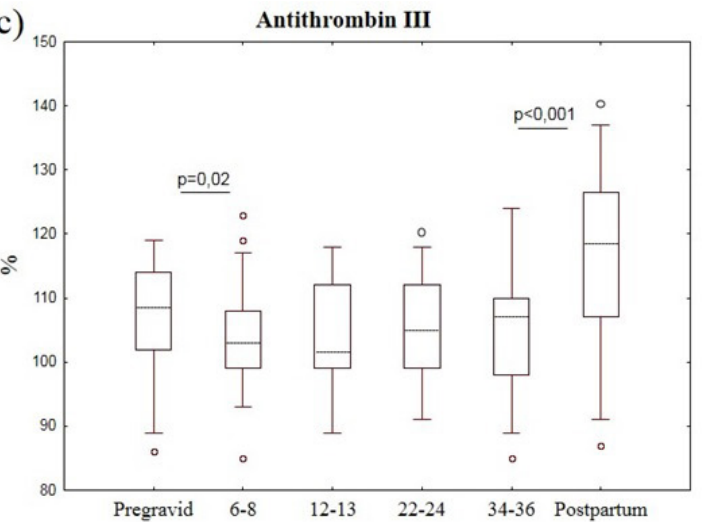

e)

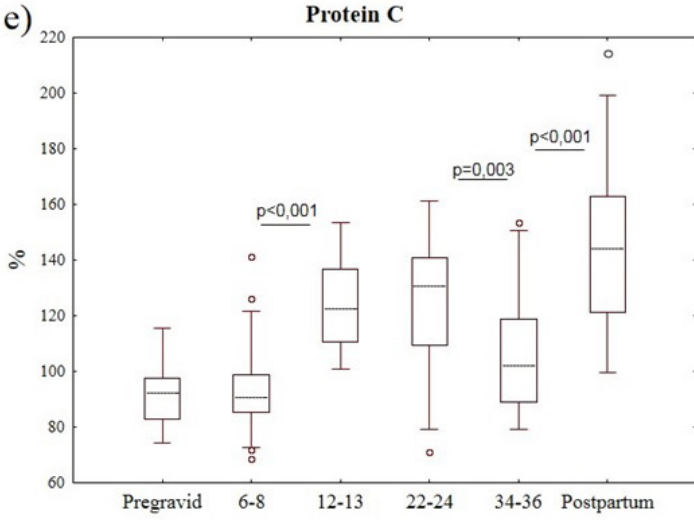

g) $)_{1,6}$ Screening for abnormalities of protein $\mathbf{C}$ system

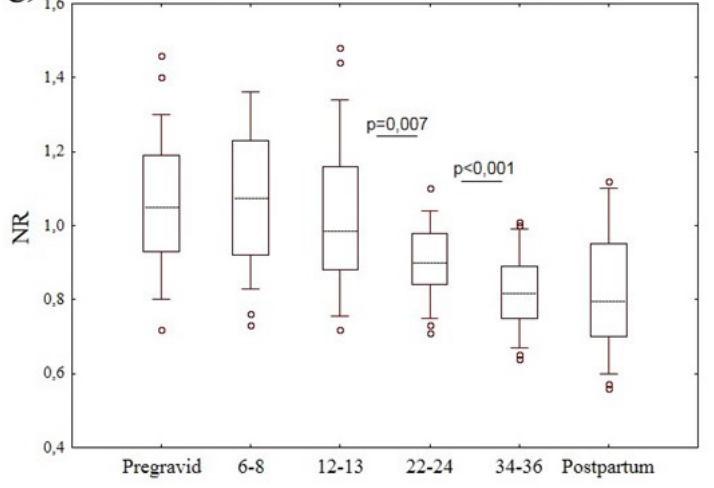

b)

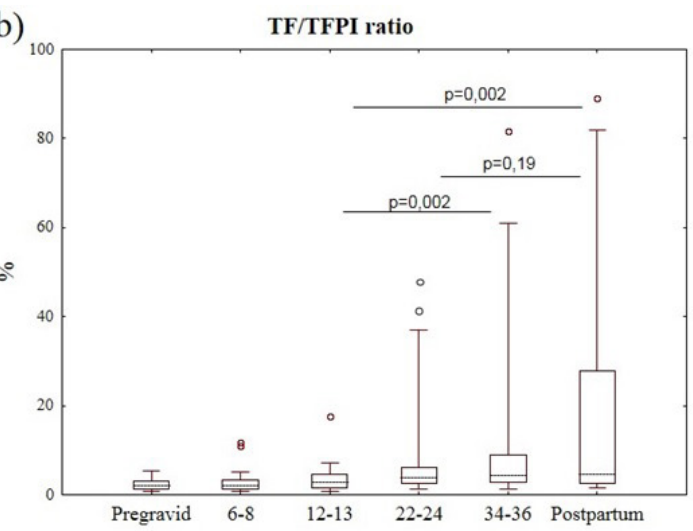

d)

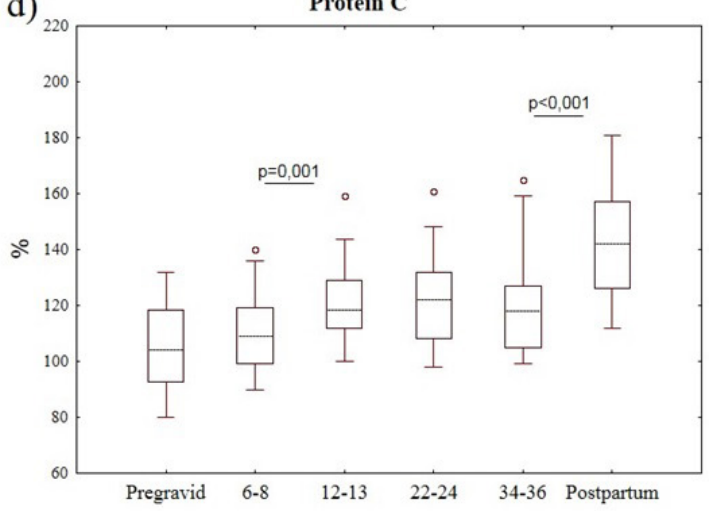

f)

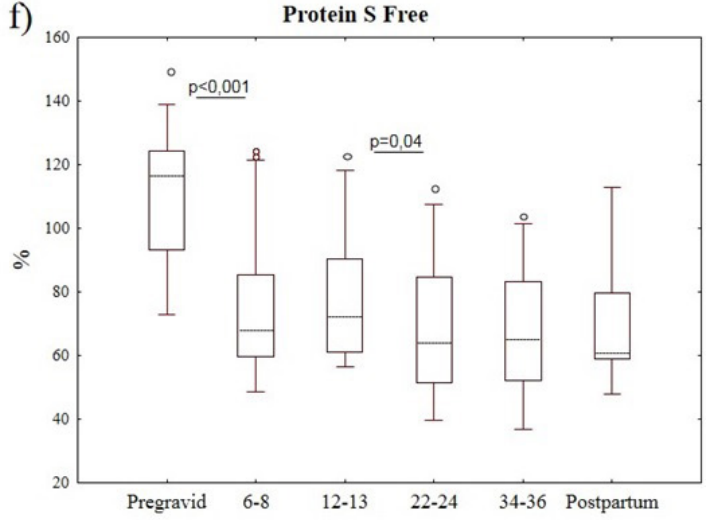

h) Thrombomodulin (soluble receptor CD141)

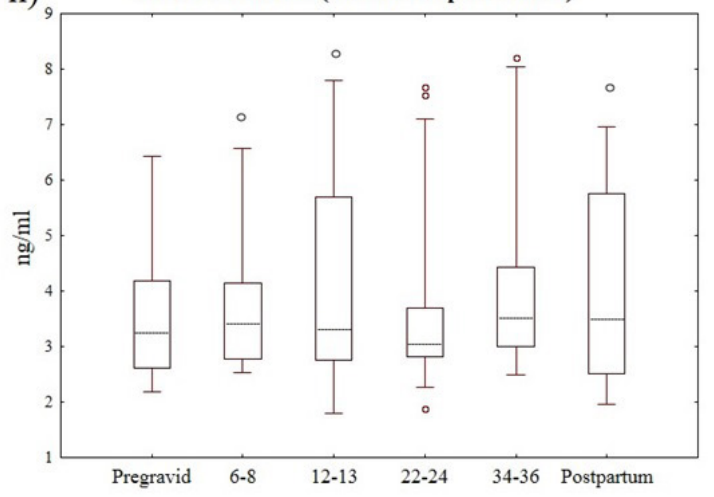

Figure 4: Box plots of reference intervals in pregravid period, different stages of pregnancy and after delivery for a) tissue factor pathway inhibitor; b) TF/TFPI ratio; c) antithrombin III; d) protein C (reagent-«Berichrom Protein C»); e) Protein C (reagent-«Protein C Reagent COAG»); f) protein S free; g) screening for abnormalities of protein $\mathrm{C}$ system; h) thrombomodulin (soluble receptor CD141). 
Citation: Momot AP, Semenova NA, Belozerov DE, Trukhina DA, Kudinova IY (2016) The Dynamics of the Hemostatic Parameters in Physiological Pregnancy and After Delivery. J Hematol Blood Transfus Disord 3: 005

a)

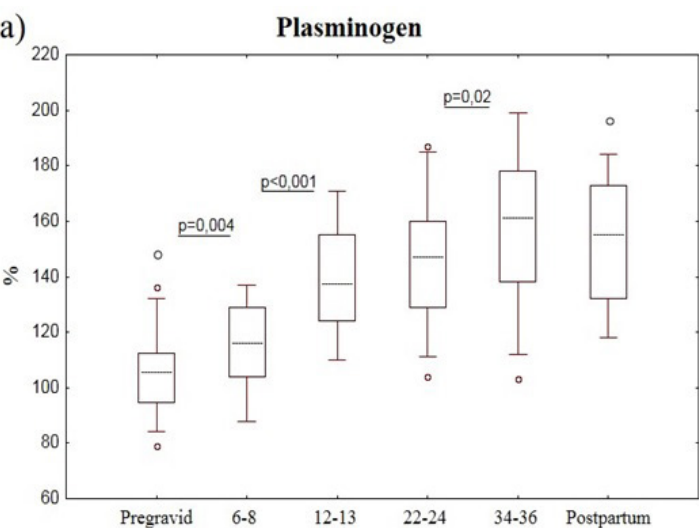

c)

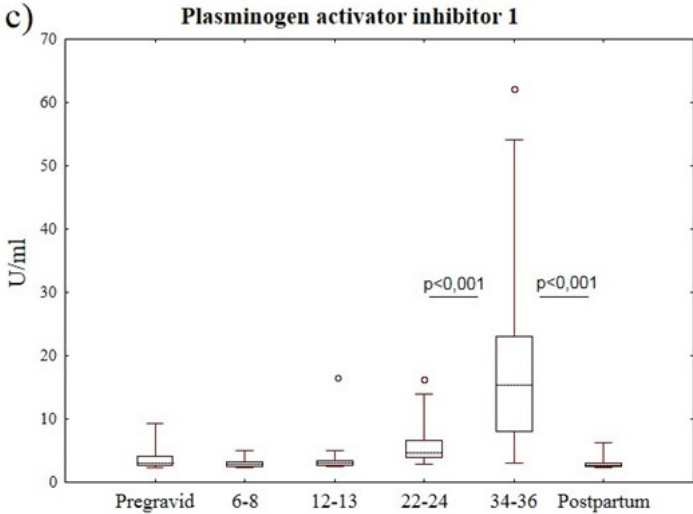

e)

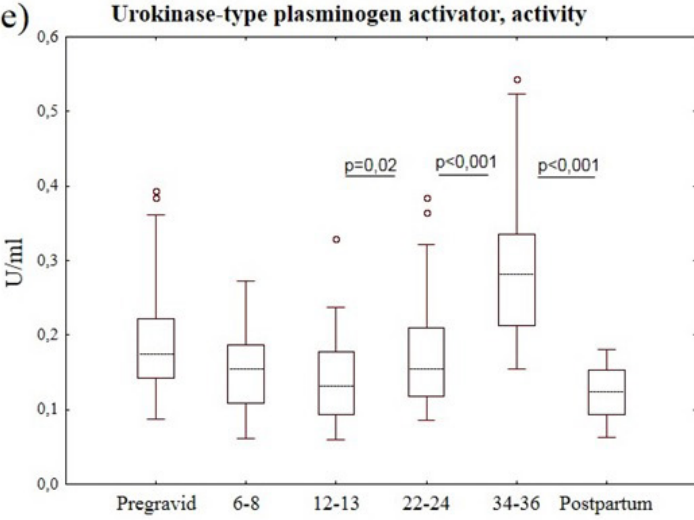

g)

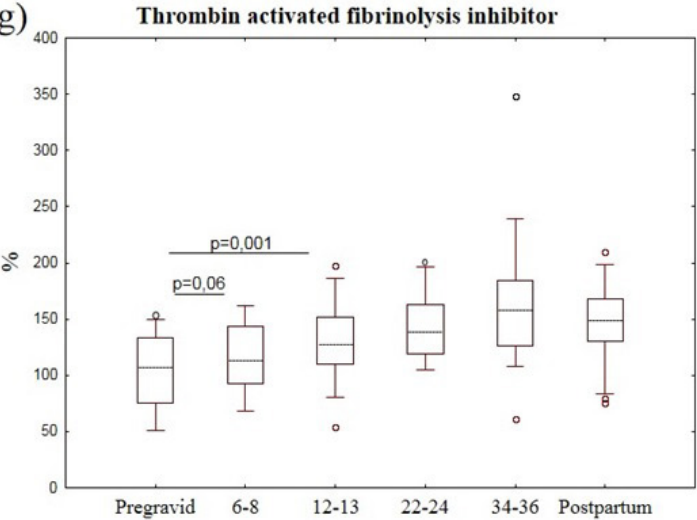

b)

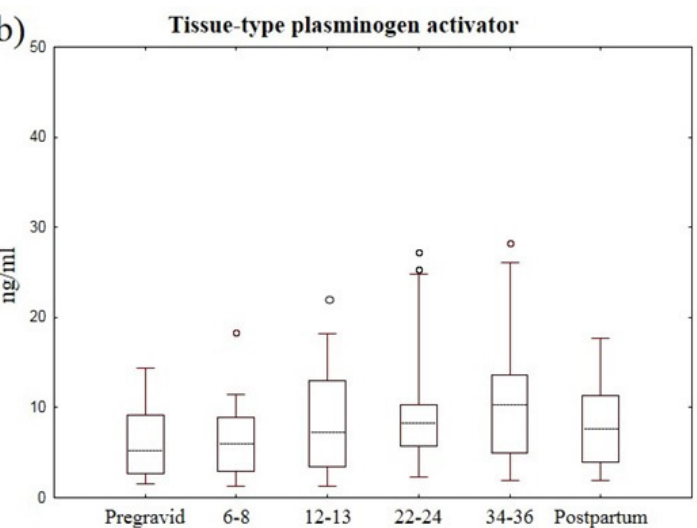

d)

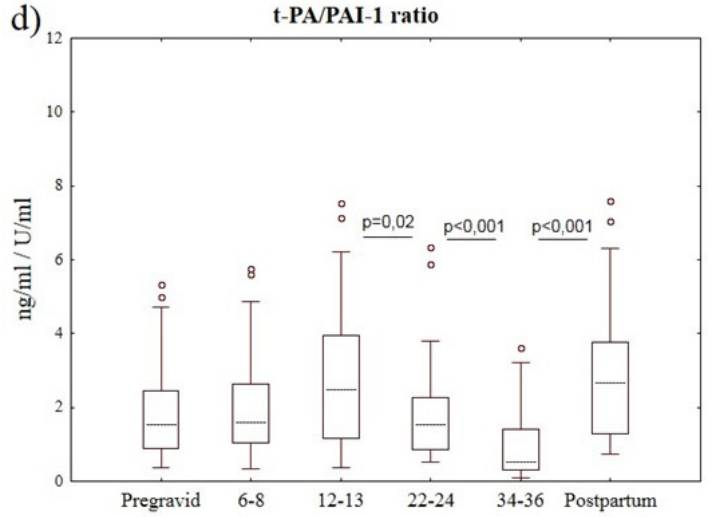

f) Urokinase-type plasminogen activator, level

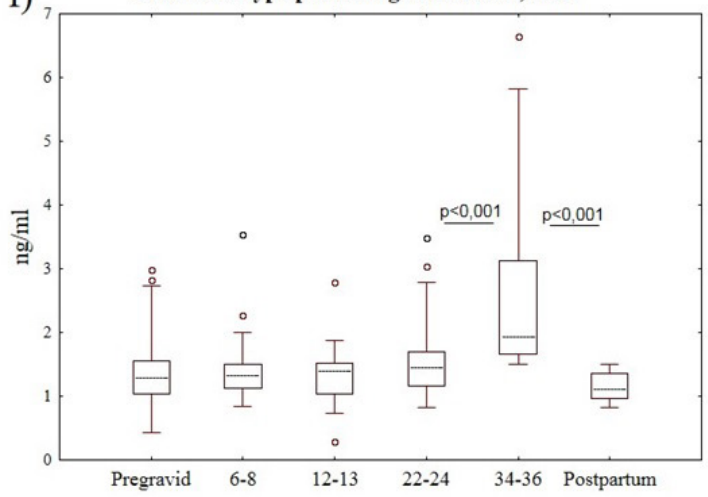

h)

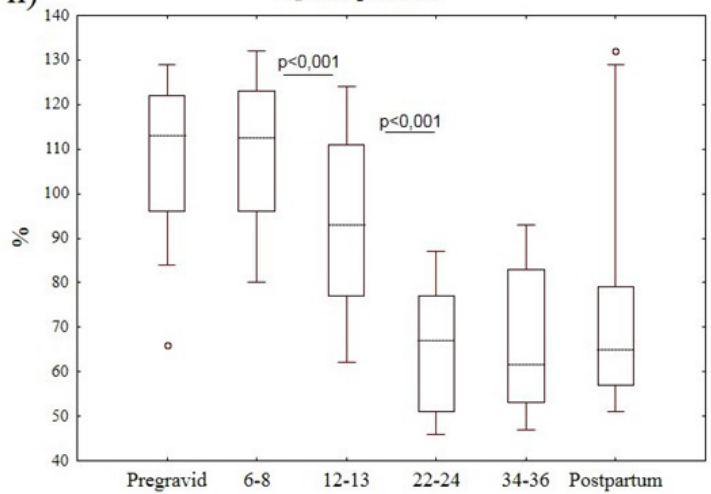

Figure 5: Box plots of reference intervals in pregravid period, different stages of pregnancy and after delivery for a) plasminogen; b) tissue-type plasminogen activator; c) plasminogen activator inhibitor 1; d) t-PA/PAl-1 ratio; e) urokinase-type plasminogen activator, activity; f) urokinase-type plasminogen activator, level; g) thrombin activated fibrinolysis inhibitor; h) $\alpha_{2}$-antiplasmin. 
women enrolled into the study received folic acid (during first 20-32 weeks of pregnancy in the dose of not less than $400 \mathrm{mg}$ per day).

\section{Coagulation factor changes}

Activity (content) of blood coagulation factors had multidirectional dynamics in the study at different stages of pregnancy. Fibrinogen concentration as well as the activity of Factors VII, VIII, X and XII were increasing consistently during pregnancy (Figures $2 \mathrm{a}, 2 \mathrm{~d}-\mathrm{g}$ ) by 1.74-3.56 times versus pregravid values regardless hemodilution specific to this period of women's life. The highest parameters were fibrinogen concentration as well as the activity of Factors VII and VIII. At the same time the increase of Factor II activity occurred only before 12-13 weeks of pregnancy (from 108 to $140 \mathrm{u} / \mathrm{dl}$ ). Later its activity remained stable including late pregnancy and first days after delivery (Figure 2b). Interesting dynamics was observed in Factor $\mathrm{V}$ (Figure 2c). This factor was stable in the first trimester of pregnancy, however, its activity decreased from $68 \mathrm{u} / \mathrm{dl}$ (12-13 weeks) to $46 \mathrm{u} / \mathrm{dl}$ (22-24 weeks). Later, the decrease of this factor activity changed into the increase reaching the pregravid values. In contrast to all other blood coagulation factors, Factor XIII was decreasing its activity consistently from 12-13 weeks of pregnancy up to delivery (Figure 2h).

Fibrinogen concentration in the study was measured with the help of two different reagents by the same manufacturer (Table 1). There was a significant correlation of the obtained data, concerning this parameter, and correlation coefficient was $0.87(\mathrm{p}<0.001)$. It should also be noted that Factor IX activity was not studied because of some problems at preanalytical phase of the study.

\section{Markers of coagulation activation}

According to the results of the study, TF activity increases starting from early pregnancy (Figure $3 \mathrm{a}$ ). As gestation progresses, the increase of TF activity was observed compared to the baseline values (pregravid period) by 1.26, 2.32 and 2.75 times at 12-13, 22-24 and 34-36 weeks of pregnancy, respectively. Trauma related to spontaneous labor was associated with the maximum activity of this key activator of coagulation, which reached the level of more than $200 \mathrm{pmol} / \mathrm{l}$ in three women out of 44 (in $6.8 \%$ of observations).

Taking into account the data mentioned above, similar dynamics of activated Factor VII (FVIIa) production might be expected. Indeed, the activation of this key coagulation factor occurred in our studies (Figure 3b). The level of Factor VIIa was increasing abruptly starting from 12-13 weeks of pregnancy (by $41.1 \%$ in comparison to the pregravid values) and remained stably high till delivery and 2-3 days after it. In relation to the results of calculations, correlation coefficient between TF and FVIIa was $0.21(\mathrm{p}<0.002)$.

The presence of TF in blood, according to up-to-date scientific opinion, leads to thrombin potential. In the study thrombin potential was measured with the help of calibrated thrombogram technology suggested by Hemker et al. [12,13]. With the help of this method, acceleration of time to reach Peak thrombin (ttPeak) generation in blood was identified in women during pregnancy (Figure 3c) as well as changes of two other parameters were used to access intensity of thrombin generation-peak thrombin and Endogenous Thrombin Potential (ETP) (Figures 3d and e).

Since early pregnancy (6-8 weeks) the latter two parameters were increasing (compared to pregravid period for Peak thrombin by $55.1 \%$ and for ETP by 39.6\%) and correlated well with each other during the whole course of pregnancy (correlation coefficient $=0.80 ; \mathrm{p}<0.001$ ).
Realization of hemostatic and, consequently, fibrinolytic reactions of hemostasis is often stated by the increase of D-dimers level in blood plasma [14]. In the present paper the content of D-dimers was determined with the help of test-systems "Auto Blue D-dimer 400», «Auto Red D-dimer 700», «IMMULITE 2000 D-Dimer» and «D-dimer Nycocard» (Table 1). Used reagents were different in their specificity and sensitivity due to the use of different monoclonal antibodies in them to epitopes of $\gamma$-chains in $\mathrm{D}$-domains of a fibrin molecule (respectively, MA8D3 are the first two reagents, DD5 and $54 \mathrm{H} 9$ ) and different determination laboratory techniques. Common in these two studies was the progressing increase of D-dimers level as gestation was progressing. However, there were significant differences between the obtained reference intervals using different reagents (Table 1, Figures $3 \mathrm{f}-3 \mathrm{~h}$ ). It should be noted that the best comparability of the results was obtained with reagents «Auto Blue D-dimer 400» и «Auto Red D-dimer 700» by the same manufacturer. Correlation coefficient between the results obtained with the help of the reagents mentioned above (Figures $3 \mathrm{f}$ and $\mathrm{g}$ ) was $0.84(\mathrm{p}<0.001)$. Moreover, the presented results show that the use of a snap analysis (Figure $3 \mathrm{~h}$ ) to examine pregnant women with the minimal interval between the values of $100 \mathrm{ng} / \mathrm{ml}$ give little information.

According to the performed analysis, the correlation was stated between the content of $\mathrm{D}$-dimers in blood plasma (with reagent «Auto Red D-dimer 700») and TF ( $\mathrm{r}=0.40 ; \mathrm{p}<0.001)$, FVIIa ( $\mathrm{r}=0.22$; $\mathrm{p}=0.001$ ) as well as with the values of thrombin generation test-peak thrombin $(\mathrm{r}=0.33 ; \mathrm{p}<0.001)$ and $\operatorname{ETP}(\mathrm{r}=0.32 ; \mathrm{p}<0.001)$.

\section{Inhibitors of blood coagulation}

Physiological blood coagulation inhibitors are numerous and aimed at controlling expressiveness of clotting reactions at different phases of this process. In this study the known parameters were studied which reflect the functioning of the most important anticoagulants during pregnancy.

Tissue Factor Pathway Inhibitor (TFPI) activity increased during pregnancy but less intensively compared to the increase of TF activity (Figures $3 \mathrm{a}$ and $4 \mathrm{a}, \mathrm{b}$ ). Significant increase of this starting coagulation inhibitor was identified only at 34-36 weeks of pregnancy and after delivery (by $24.0 \%$ and $72.2 \%$, respectively, compared to the results of pregravid women).

Antithrombin III activity was not significantly changed (Figure 4c); however, there were significant changes in anticoagulant system connected with protein C. In the study protein C activity was measured with the help of two different reagents by the same manufacturer (Table 1 and figures $4 \mathrm{~d}, \mathrm{e}$ ). According to the obtained results, the increase of protein C activity from 12-13 weeks to 22-24 weeks of pregnancy was identified. Later, before delivery the activity of this anticoagulant was decreasing and it was more noticeable with coagulation method of the study (Figures 4e). The decrease is followed by the significant increase of protein $\mathrm{C}$ activity in the first days after delivery. It should be noted that correlation coefficient between the data obtained with the help of coagulometric and amidolytic analysis of protein $C$ was $0.66(\mathrm{p}<0.001)$.

Protein C cofactor-protein S had completely different dynamics during pregnancy that significantly increased anticoagulant and profibrinolytic activity of protein C. According to the performed studies, the activity of protein S Free was decreasing from 6-8 weeks of pregnancy (Figure 4f) by $42.1 \%$ compared to women in pregravid period and this decrease remained stable till the first days after delivery. 
The found changes were accompanied by the decrease of Normalized Ratio (NR) in the test screening for abnormalities of protein C system from 12-13 weeks of pregnancy (Figure $4 \mathrm{~g}$ ) and by the stable content of free thrombomodulin in blood plasma (Figure 4h).

\section{Fibrinolysis}

Important events during pregnancy were connected with the participants of fibrinolytic system of blood determining the limitation of enzymatic reactions of blood coagulation and fibrin.

The present study stated the dynamic increase of plasminogen level during pregnancy (Figure 5a). Plasminogen concentration growth was seen in early pregnancy (6-8 weeks) and its level increased (compared to the pregravid data) by $12.6 \%, 56.3 \%, 57.9 \%$ and $68.5 \%$ at 6-8 weeks, 12-13 weeks, 22-24 weeks and 34-36 weeks of pregnancy, respectively.

The level of t-PA remained stable during the whole pregnancy (Figure 5b), however its competitor Plasminogen Activator Inhibitor 1 (PAI-1) showed "explosive growth" of concentration by 1.5 and 5.0 times (from the baseline level to the onset of pregnancy) in the second half of pregnancy at 22-24 and 34-36 weeks, respectively (Figure 5c). After delivery the level of this inhibitor returned to the baseline (pregravid) values, decreased significantly by $82 \%$ compared to the values at 34-36 weeks of pregnancy.

In our study t-PA/PAI-1 ratio was also counted concerning their contest for plasminogen activation. The domination of PAI-1 level over t-PA level in the second half of pregnancy (according to the calculated ratio) is well seen in figure $5 \mathrm{~d}$.

Another important plasminogen activator-urokinase-type Plasminogen Activator (u-PA) increased significantly during pregnancy starting from 22-24 weeks and the value of this parameter (both activity and concentration) was the highest in late pregnancy (Figures $5 \mathrm{e}$ and $\mathrm{f}$ ). It was also found that quantitative characteristics of $\mathrm{u}$-PA were decreased in the first days after delivery reaching the pregravid values.

Thrombin Activated Fibrinolysis Inhibitor (TAFI) content increased in blood plasma from early pregnancy in women. Particularly, the growth of its level was identified at 6-8 weeks by $5.9 \%$ (compared to pregravid values), at $12-13$ weeks by $18.8 \%$, at $22-24$ weeks by $29.2 \%$ and in late pregnancy by $47.4 \%$ (Figure $5 \mathrm{~g}$ ).

According to the obtained data the $\alpha_{2}$-AP decreased progressively during pregnancy (Figure $5 \mathrm{~h}$ ). Since 12-13 weeks of pregnancy $a_{2}$-AP was lower by $17.7 \%$ compared to pregravid values. Later this parameter continued to decrease (by $40.7 \%$ (22-24 weeks) and $45.6 \%$ (34-36 weeks). First 2-3 days after spontaneous labor $\alpha_{2}$-AP activity had the tendency to the increase; however there was a wide range of data in sample data.

\section{Discussion}

\section{Part 1}

In the present study prothrombotic manifestations in vascular-platelet hemostasis include the increase of platelet aggregation activity in response to low dose of ADP $(0.1 \mu \mathrm{mol})$, the increase of RCoA and vWF Ag with the simultaneous decrease of a potential of metalloproteinase ADAMTS-13. During physiological pregnancy platelet activation and the increase of their ability of aggregation were described earlier. The latter was explained by the increase of a number of aggregates of circulating platelets as well as by the increase of a basal level of a component of platelet alpha granules CD63 $[15,16]$. There is a study by Argentinian researchers devoted to the study of ADAMTS-13 activity versus vWF antigen in blood plasma during physiological pregnancy [17] based on earlier studies by Mannucci et al., [18]. According to this publication, including observations of 325 women at the age from 14 to 41 , the decrease of ADAMTS-13 activity was registered in up to $65 \%$ of normal range in pregravid period (17-23 weeks), 61\% (24-28 weeks), 65\% (29-35 weeks), 58\% (36-40 weeks), and $52 \%$ (the first two days after delivery). Besides, in their study the dependence between the increase of vWF antigen and the decrease of the amount of enzyme ADAMTS-13 (Pearson correlation coefficient-0.22, $\mathrm{P}=0.0003$ ) was identified during pregnancy. It is suggested that the increase of vWF Ag level during pregnancy is connected with hyperestrogenemia and activation of its synthesis by endothelium of blood vessels $[9,19]$.

VWF is known to be a complex multimeric adhesive glycoprotein (in monomeric form $280 \mathrm{kDa}$ ) synthesized by endothelial cells and megalokaryocytes [20]. A special role of this factor in hemostatic system is determined by its structure in the form of a series of heterogeneous multimers (from 0.5 to $20 \mathrm{mln} \mathrm{Da}$ ) consisting of repetitive subunits with binding domains of glycoprotein platelet receptors (GPIb and IIb/IIIa), collagen, heparin, and Factor VIII blood coagulation. It mediates platelet adhesion to subendothelium through the interaction with platelet receptor-glycoprotein Ib [21]. Large vWF multimers are cleaved by metalloproteinase ADAMTS- 13 during their release from endothelial cells under the influence of proinflammatory cytokines [19].

Ultra large vWF multimers with low activity of ADAMTS-13 initiate platelet activation and are able to lead to thrombocytopenia and multiple formation of hyaline (thrombocytic) thrombi in microcirculation vessels in Thrombotic Thrombocytopenic Purpura (TTP) $[11,20]$. It can be stated that vWF activity during pregnancy is in opposition to functional activity of metalloproteinase ADAMTS-13 released by endothelium of blood vessels and cleaving vWF. Failure to degrade large vWF multimers is associated with inherited and idiopathic types of TTP or predispose to similar abnormalities [22]. TTP is more common in women $(66-78 \%)$ and $12-25 \%$ of the episodes of this abnormality are related to pregnancy [23] mostly in the third trimester and in postpartum period [24,25].

The work by Lattuada A et al., is of great interest, it describes the reduction of the enzyme ADAMTS 13 activity in patients with HELLP-syndrome (31\% ranging 12-43\%) compared to women in the third trimester of pregnancy (71\% ranging $48-105 \%)$ and nonpregnant women (101\% ranging 45-152\%) [26]. ADAMTS13 activity often falls below threshold level in antiphospholipid syndrome [27]. Besides, the connection between the decrease of ADAMTS-13 activity and the increase of RCoA and vWF antigen in pregnant women with pre-eclampsia has recently been shown [28]. In general, it can be assumed that the increase of RCoA and vWF level is not that significant for pregnancy as the decrease of ADAMTS-13 activity and antigen potentially contributing to the increase of this factor multimerization and increase of platelet activation reaching delivery.

In our study, the decrease of homocysteine level was determined during pregnancy reaching its baseline values at first days after delivery, which was also described in previous publications $[29,30]$. According to our results, coefficient correlation between platelet activation (ADP in dose $0.1 \mu \mathrm{mol} / \mathrm{l}$ ) and the level of homocysteine was $0.37(\mathrm{P}<0.001)$. It is suggested that the increase of its level more than 
$15 \mu \mathrm{mol} / \mathrm{l}$ may lead to platelet activation and stimulation of Tissue Factor (TF) expression and, consequently, to the risk increase of pregnancy complications [31].

\section{Part 2}

Similar dynamics of activity of a number of blood clotting factors (fibrinogen, Factors VII, VIII, X, and XIII) compared to the presented data during pregnancy was described earlier by many authors. In contrast with our results, Szecsi et al., [9] obtained the data about the relatively stable levels of activity of blood clotting Factors II, V, X, and XII, which stayed almost unchanged during pregnancy, delivery and postpartum period and were within reference range in nonpregnant women. In earlier studies, however, the increase of Factor X activity was shown in pregnant women compared to the control group [32-34]. In our study there is no confirmation of the decrease of Factor XI activity $[1,35]$. Besides, there are some differences related to the Factor V activity. Some authors [9] state that Factor V activity is stable during pregnancy and delivery, others [36] evidence the gradual increase of this factor during pregnancy.

The decrease of Factor XIII in the second half of pregnancy is mentioned in these studies. Earlier studies showed the increase of this factor at the beginning of pregnancy and return to normal values in the third trimester for nonpregnant women [37]. The decrease of Factor XIII seems to be associated with its participation in fibrin stabilization, massive formation of which is more likely in late physiological pregnancy due to the growth of $\mathrm{D}$-dimers. More detailed data on the characteristics of activity of blood clotting factors and other parameters of hemocoagulation can be found in the reviews [7,38-41].

\section{Part 3}

A lot of studies are devoted to the process of blood coagulation activation during pregnancy, in contrast there are few studies devoted to the initiation of blood coagulation at this period of woman's life. The obtained data showed the regular increase of TF activity in blood plasma as gestation progresses and due to it the increase of blood coagulation activity is at the peak of its expressiveness in the period close to delivery accompanied by placental expulsion [42]. As a strong initiator of coagulation TF, predominantly of placental origin $[43,44]$, leads to thrombosis of vessels quickly to stop bleeding in women during delivery. It is very important since massive bleeding might occur in placental bloodstream $700 \mathrm{ml} / \mathrm{min}^{-1}$ because of coagulation system failure [8]. At the same time, the reasonability of the significant increase of the parameter as well as Factor VIIa level from 12-13 weeks of pregnancy compared to nonpregnant women is not clear in our study since delivery and consequently limitation of the extent of blood loss in the first half of pregnancy are not programmed by nature. Meanwhile, in comparison with the values of pregnant women, the mean concentration of TF in mother's plasma is higher in patients with preeclampsia or with preterm prelabor rupture of membranes $[45,46]$. An interesting suggestion was made about the connection of excessive expression of TF to bloodstream and the onset of preterm labor [47] that we share completely.

TF is an active initiator of coagulation system that can initiate thrombin generation. In this regard, our data do not contradict to previously published results by other authors devoted to the assessment of peculiarities of thrombin generation test during pregnancy $[8,48,49]$. The test for the assessment of disposable thrombography records the final result of the complex interaction of enzymes involving in blood coagulation. According to McLean et al., [8] and some other below mentioned authors, the measurement of individual blood plasma capacity to generate thrombin under the TF action might become the best indicator of thrombotic readiness compared to other tests aimed at the study of fibrin clots formation or determination of potential biomarkers-prothrombin fragment $1+2$, fibrinopeptide A, thrombin-antithrombin complex, and D-dimers $[50,51]$.

Manifestations of thrombotic readiness of blood during pregnancy is traditionally explained by the increase of $\mathrm{D}$-dimers level presenting the result of a sequential intervention on thrombin fibrinogen, activated Factor XIII and plasmin $[34,52,53]$. In clinical practice the increase of $\mathrm{D}$-dimers concentrations is widely used as laboratory marker of blood coagulation activation in such human pathologies as Disseminated Intravascular Coagulation (DIC) [54,55], deep vein thrombosis of lower extremities and pulmonary embolism $[14,56]$. However, a question arises in the assessment of informational content and clinical significance of determination of D-dimers during pregnancy to exclude Venous Thrombo Embolic Complications (VTEC). Some authors discredit the informational content of D-dimers level determination of (from the point of view of diagnostics of venous thromboembolic complications) due to the natural growth of its concentration as gestation progresses without any types of pathologies $[9,57,58]$. Anyway the question about the cutoffs of D-dimers of venous thromboembolic complications at different stages of pregnancy is being discussed [53,59]. We will return to the question about the origin of $\mathrm{D}$-dimers during physiological pregnancy after the discussion of fibrinolytic activity of blood in this period of women's lives.

\section{Part 4}

Physiological anticoagulants including TFPI, antithrombin III, and protein $\mathrm{C}+\mathrm{S}$ system resist uncontrolled blood coagulation. In our study the increase of TFPI level was not found in blood plasma in women in the first half of pregnancy, which was determined by Sarig et al., [60]. However, our data correspond to the results published by Uszynski et al., [44] who determined marked increase of TFPI activity during delivery and first days after this event.

Antithrombin III remains stable during pregnancy and delivery in women [9], which is consistent with our data and the results of studies by other authors $[34,36,61]$. In contrast, literature data on the concentration of protein $\mathrm{C}$ during physiological pregnancy are controversial. In the studies by other authors, in contrast to our data, no significant dynamics of protein $\mathrm{C}$ level was observed during the whole period of pregnancy $[40,62,63]$. Kjellberg et al., [64] found the increase of the concentration of this anticoagulant in the second trimester of pregnancy. However, a significant decrease of this parameter was demonstrated at 35-40 weeks of pregnancy in another study [34]. Protein C cofactor-protein S enhances its anticoagulant and profibrinolytic effect of protein C. As it is stated, the values for both total protein $S$ and free protein $S$ go below the normal range very early and mainly in late pregnancy $[9,34,63]$, which corresponds with our data. Although the fall in free protein $S$ level during pregnancy is a physiological event, it is unclear whether it contributes to the hypercoagulable state of pregnancy and the increased incidence of thromboembolism [39]. It should be noted that the increase of thrombomodulin level, earlier observed in pregnancy, was not found in this study $[65,66]$. 


\section{Part 5}

Fibrinolytic reactions are also an important obstacle to excessive coagulation enhancing the effect of physiological anticoagulants. The key enzyme of fibrinolysis is plasmin, which is formed from the inactive proenzyme-plasminogen under control of the activators ( $\mathrm{t}-\mathrm{PA}, \mathrm{u}-\mathrm{PA}$ ) and inhibitors of this process (PAI-1, PAI-2 (plasminogen activator inhibitor 2), appearing during pregnancy, TAFI). Besides, plasmin in blood circulation can quickly lose its activity due to the formation of inactive complex with $\alpha_{2}$-AP. These features make the fibrinolytic system of blood similar to hemocoagulation system in complexity although there are a lot of differences.

Some studies consider that fibrinolytic activity of blood plasma decreases in pregnancy and stays at the low level during and after delivery and normalizes after delivery. These statements are based on the fact-finding of prolongation of clot lysis time of fibrin that was collected from blood plasma $[67,68]$, the decrease of t-PA concentration, as well as on the increase of PAI-1 and PAI-2 levels $[61,69,70]$. Some authors presuppose that the observed changes earlier viewed activators and inhibitors do not influence the general fibrinolytic activity of blood [71,72]. However, it contradicts to the evident and significant increase of D-dimers concentrations in blood as gestation progresses [73]. Considering the latter, as well as the increase of concentration of plasmin- $\boldsymbol{\alpha}_{2}$-AP complex, other authors think that fibrinolytic activity during pregnancy is not decreased but increased compared to pregravid women [32,34]. Consequently, there is no common opinion and it is not clear why thrombosis are absent although D-dimers increase, especially, in late pregnancy. The excessive formation and degradation of fibrin is considered to take place locally in uteroplacental blood flow by some scholars [74]. However, without accepting the above mentioned reasons for the increase of D-dimers level during pregnancy, we will express our opinion about their origin in this physiological state.

To comment our results related to fibrinolytic system of blood we will note that Kruithof et al., [71] measured plasminogen by rocket immunoelectrophoresis did not find significant differences between pregnant and nonpregnant women. Nevertheless, the increase of plasminogen concentration during pregnancy, that we registered, was described by other authors in more recent studies [34,75]. In the study by Uchikova et al., [34] positive dynamics of plasminogen concentration is suggested to be connected with both hyperproduction of the proenzyme and its decreased use during pregnancy. These authors think that this change of plasminogen level might be considered as a protective reaction decreasing the probability of thrombus formation.

In contrast to our obtained data, in earlier published studies, the decrease of t-PA activity in blood plasma during pregnancy was determined [70] and later publications report about the moderate increase of this parameter in pregnant women without any marked pathologies [76,77]. It should be noted that the decrease of t-PA level and activity is connected with an increased risk of thrombosis [78], as well as with intrauterine growth retardation and preeclampsia [79].

Together with t-PA, urokinase-type Plasminogen Activator (u-PA) can be referred to the strong physiological plasminogen activators. Normally, it accounts for $15 \%$ of the potential of blood and blood vessel walls that activates fibrinolysis [71]. This plasminogen activator is synthesized by juxtaglomerular apparatus of kidneys, endotheliocytes, fibroblasts, epithelial cells, as well as decidual cells of placenta and becomes active after the connection with a receptor uPAR/CD87 of monocytes, fibroblasts and other cells of intravascular space [80]
The presence of receptors to $\mathrm{u}-\mathrm{PA}$ in many cells allow this plasminogen activator to manifest its activity not only close to blood vessel walls but also in intercellular space of systemic circulation.

Our results in the assessment of u-PA dynamics were similar to the results by other authors. Saleh et al., [81] gave the following range of $\mathrm{u}-\mathrm{PA}$ concentrations during pregnancy: in the first trimester-median $0.9 \mathrm{ng} / \mathrm{ml}(95 \%$ confidential interval-CI $0.6-2.8 \mathrm{ng} / \mathrm{ml})$, in the second trimester-1.5ng/ml (CI $0.6-2.2 \mathrm{ng} / \mathrm{ml})$, in the third trimester- $2.1 \mathrm{ng} / \mathrm{ml}$ (CI 0.9-6.ng/ml), during delivery-2.0ng/ml (CI 0.5-3.4ng/ml) and 1-2 days after delivery-0.6ng/ml (CI $0.2-1.3 \mathrm{ng} / \mathrm{ml}$ ). Similar results were also obtained by Koh et al., [82]. It is interesting to note that Lindoff et al., [83] found comparatively lower levels of u-PA in cases of intrauterine growth retardation and preeclampsia.

In physiological states the process of plasminogen activation is balanced by reactions of inactivation. PAI- 1 is referred to one of the most significant plasminogen activator inhibitors [84]. As t-PA, this inhibitor of fibrinolytic reactions is produced by endothelial cells of blood-vessels, hepatocytes, monocytes, macrophages, fibroblasts, muscle cells and it refers to the serpin family. PAI- 1 penetrates into the fibrin clot in concentration significantly higher than its level in blood plasma, which leads to thrombus stabilization and increases its resistance to lysis [85].

In our studies, the increase of PAI-1 level in late pregnancy is in agreement with available literature data [76,81]. In addition, earlier growth of concentration in earlier stages in preeclampsia and intrauterine growth retardation was discovered $[81,86]$. In our study the content of PAI-2 in blood in pregnant women was not studied, however, it is known that it is being increased during pregnancy and is associated with the presence and age of placenta [87]. We analyzed the dynamics of another fibrinolysis inhibitor-thrombin activated fibrinolysis inhibitor-TAFI of endothelial origin capable to destroy catalytic surface of fibrin (lysine binding site) needed for plasminogen activation with the help of t-PA. Its inactive form-procarboxypeptidase Y-is activated by thrombin-thrombomodulin complex and by a number of other agents until active carboxypeptidase $\mathrm{Y}[88,89]$. Therefore, TAFI as well as PAI-1 plays a role predominantly in parietal reactions of fibrinolysis contributing the strengthening of a fibrin clot and prevention of early lysis [43].

The increase of TAFI content in blood plasma during pregnancy according to our data corresponded with the results described earlier $[41,68,90]$, although there are some data about the increase of this inhibitor only in the third trimester [91].

In the presented study, the dynamics of the content of the direct plasmin inhibitor- $\alpha_{2}$-AP was studied. It belongs to serpin family [92] and has hepatic origin. It is the main biological plasmin inhibitor which has about $90 \%$ of antiplasmin activity [93]. $a_{2}$-AP is known to be connected with plasmin which circulates in blood with the formation of inactive plasmin- $\alpha_{2}$-antiplasmin complex [94-96]. There are different understandings related to the changes of $\alpha_{2}$-AP activity in literature. In some studies there was the increase of activity of this plasmin inhibitor between the onset of pregnancy and the end of pregnancy $[34,41]$. At the same time, the results published by Holmes and Wallace [7] evidence the significant dynamic decrease of $\alpha_{2}$-AP activity closer to delivery, which is in agreement with our results. It is interesting to note that inherited and acquired $\alpha_{2}$-AP deficiency is associated with severe bleedings due to uncontrolled hyperfibrinolysis [97]. 
In the light of the above, it is possible to suppose that the increase of $\mathrm{u}$-PA level with a sharp decrease of $\alpha_{2}$-AP activity in late pregnancy makes the optimal conditions for plasmin formation and its free circulation and, consequently, for the increase of fibrinolytic activity of blood in systemic circulation. This thesis is confirmed by the presence of correlation between $\mathrm{u}$-PA level and $\alpha_{2}$-AP activity $(\mathrm{r}=-0.168 ; \mathrm{p}=0.012)$, $\mathrm{u}-\mathrm{PA}$ level and $\mathrm{D}$-dimers concentration (reagent «Auto Red D-dimer 700») $(\mathrm{r}=0.306 ; \mathrm{p}<0.001), \alpha_{2}$-AP activity and $\mathrm{D}$-dimers concentration (the same reagent) ( $\mathrm{r}=-0.552 ; \mathrm{p}<0.001)$. In this case, the division of the function of fibrinolytic system is appeared to be according to the influence focus. Parietal (intravascular) reactions of fibrinolysis are inhibited and this fact is illustrated by the domination of PAI-1 growth compared to t-PA as well as by the increase of TAFI level. As a result, plasminogen, that has affinity to fibrin, significantly loses its capability of conversion to proteolytic active enzyme. It enhances stability of fibrin formation in vessels injured during delivery. This mechanism can be crucial for lowering the extent of blood loss in cases when a large number of injured vessels in uterus need thrombosing. Apparently, abnormality of the mechanism can lead to "hyperfibrinolytic" bleedings during delivery and in early postpartum period.

So, what is the substrate for the formation of D-dimers in late pregnancy? To find out the answer to this question, the attention should be paid to the study by Meesters et al., [98] according to which high D-dimer levels in hospitalized patients might reflect one of the processes underlying the disease which initiates intravascular fibrin formation but does not necessarily lead to evident thrombosis. Fibrin is determined to be enhanced by covalent links with the help of Factor XIIIa at the soluble stage before it converts to gel (clot) [99-101]. Thus, it is assumed that the substrate for the formation of D-dimers in certain conditions could be only not fibrin clot but also its soluble predecessors, which is discussed in sufficient detail in the review by Adam and Key [14]. In relation to it, we hypothesize that in the period before delivery, despite the increase of hemostatic potential with excessive thrombin generation, soluble forms of stabilized fibrin are primarily exposed to proteolysis by plasmin and it breaks the classical way to convert fibrinogen to fibrin clot. Besides, high D-dimers level could evidence the effectivity of protective mechanisms preventing from intravascular blood coagulation and thrombus formation before and during delivery. It can be also suggested that the decrease of plasmin potential in circulation primarily related to $\alpha_{2}$-AP is capable to increase the likelihood of venous thromboembolic complications in obstetric patients.

\section{Conclusion}

A wide range of laboratory data is studied in the paper which characterizes the peculiarities of hemostatic system at different stages of physiological pregnancy and at first days after vaginal delivery compared to the values of these parameters in pregravid period. Reference ranges of different study methods related to the hemostatic system in xanthochroid women are presented. They might be different between racial groups, regions of the world as well as due to the peculiarities of pre-analytical and analytical phases of laboratory testing. However, despite these limitations, the presented data related to the study of 50 parameters in the same blood plasma samples allow studying the evolution of hemostatic reactions during physiological pregnancy without bleedings and thromboses. Besides, separate studies of the same parameter were performed with different reagents to support the revealed consistencies related to the dynamics of the results of some tests. (von WF Ag, fibrinogen and D-dimers, protein C activity).
According to the obtained results and taking into account the opinions of other authors, we consider that:

- The assessment of the excessive platelet activation during pregnancy can be performed in the study of aggregation of these cells with minimum dose of ADP. This method along with others can be used in order to get objective reasons to prescribe antiaggregants;

- The decrease of metalloproteinase ADAMTS-13 activity and level starting from 12-13 weeks of gestation can be significant for platelet activation and risk of thrombotic microangiopathy;

- Identification of the excessive thrombin generation (in relation to reference ranges) associated with different congenial (Factor $\mathrm{V}$ Leiden mutation, prothrombin mutation) or acquired thrombogenic risk factors can be crucial for the decision making whether to prescribe heparin prophylaxis during pregnancy. Currently we have got evidence and experience in this field on the assessment of the effectivity of low molecular weight heparin usage in women with excessive thrombin generation in ovarian stimulation in in vitro fertilization cycle [102];

- High TF level in combination with excessive thrombin generation (for parameters Peak thrombin, Endogenous Thrombin Potential (ETP)) exceeding the reference values in late pregnancy might initiate not only intravascular blood coagulation but also the end of pregnancy and the onset of labor. In this case, the likelihood of fatal blood loss for the mother is decreased. The mentioned peculiarities of hemostatic system might be directly relevant to the onset of preterm labor;

- There is a mechanism that has not been described earlier. It prevents thrombus formation in late pregnancy and is associated with the enhancement of fibrinolytic reactions in systemic circulation, which promote fibrin degradation (with the formation of D-dimers) before its conversion into gel or fibrin clot;

- The obtained data can be used as indicative to develop the criteria for the formation of groups of women at risk for thrombotic, hemorrhagic, and obstetric complications during pregnancy and delivery.

In general, hemostatic balance seems to be supported by counteraction of coagulation and fibrinolysis. At the present time, we are not ready to give a list of informative methods to study hemostatic system in pregnant women to identify pathological states timely. We consider that further studies are needed aimed at studying the causes of a shift in the hemostatic balance in various types of pregnancy pathologies.

\section{Acknowledgement}

The authors greatly appreciate the help of Romanov VV, Fadeeva NI, Tsyvkina LP and Sidorenko AA for the obtainment of original data and preparation of this article to publication. The study is carried out at the expense of the grant RFFI № NK 14-04-98005 and supported by the company "Technology-Standard” Ltd. (Russia). The authors stated that there are no conflicts of interest regarding the publication of this article.

\section{References}

1. Brenner B (2004) Haemostatic changes in pregnancy. Thromb Res 114: 409-414.

2. Franchini M (2006) Haemostasis and pregnancy. Thromb Haemost 95: 401413. 
Citation: Momot AP, Semenova NA, Belozerov DE, Trukhina DA, Kudinova IY (2016) The Dynamics of the Hemostatic Parameters in Physiological Pregnancy and After Delivery. J Hematol Blood Transfus Disord 3: 005

3. Braekkan SK, Mathiesen EB, Njølstad I, Wilsgaard T, Hansen JB (2010) Hematocrit and risk of venous thromboembolism in a general population. The Tromso study. Haematologica 95: 270-275.

4. Heit JA (2013) Thrombophilia: Clinical and Laboratory Assessment and Management. In: Kithens CS, Konkle BA, Kessler CM (ed.). Consultative Hemostasis and Thrombosis. (3rdedn), Elsevier Saunders, Philadelphia, USA. Pg: 205-239.

5. de Lange NM, Lancé MD, de Groot R, Beckers EA, Henskens YM, et al. (2012) Obstetric hemorrhage and coagulation: an update. Thromboelastog raphy, thromboelastometry, and conventional coagulation tests in the diagnosis and prediction of postpartum hemorrhage. Obstet Gynecol Surv 67: 426-435.

6. Abbassi-Ghanavati M, Greer LG, Cunningham FG (2009) Pregnancy and laboratory studies: a reference table for clinicians. Obstet Gynecol 114: 1326-1331.

7. Holmes VA, Wallace JM (2005) Haemostasis in normal pregnancy: a balancing act? Biochem Soc Trans 33: 428-432.

8. McLean KC, Bernstein IM, Brummel-Ziedins KE (2012) Tissue factor-dependent thrombin generation across pregnancy. Am J Obstet Gynecol 207: 135.

9. Szecsi PB, Jørgensen M, Klajnbard A, Andersen MR, Colov NP, et al. (2010) Haemostatic reference intervals in pregnancy. Thromb Haemost 103: 718727.

10. Harrison P, Mackie I, Mumford A, Briggs C, Liesner R, et al. (2011) Guidelines for the laboratory investigation of heritable disorders of platelet function. $\mathrm{Br} J$ Haematol 155: 30-44.

11. Key N, Makris M, O'Shaughnessy D, Lillicrap D (2009) Practical Hemostasis and Thrombosis. (2ndedn), New Jersey, Wiley Blackwell Publishing Ltd, UK.

12. Hemker HC, Giesen P, Al Dieri R, Regnault V, de Smedt E, et al. (2003) Calibrated automated thrombin generation measurement in clotting plasma. Pathophysiol Haemost Thromb 33: 4-15.

13. Hemker HC, Al Dieri R, De Smedt E, Béguin S (2006) Thrombin generation a function test of the haemostatic-thrombotic system. Thromb Haemost 96 553-561.

14. Adam SS, Key NS, Greenberg CS (2009) D-dimer antigen: current concepts and future prospects. Blood 113: 2878-2887.

15. O'Brien WF, Saba HI, Knuppel RA, Scerbo JC, Cohen GR (1986) Alterations in platelet concentration and aggregation in normal pregnancy and preeclampsia. Am J Obstet Gynecol 155: 486-490.

16. Holthe MR, Staff AC, Berge LN, Lyberg T (2004) Different levels of platele activation in preeclamptic, normotensive pregnant, and nonpregnant women. Am J Obstet Gynecol 190: 1128-1134.

17. Sánchez-Luceros A, Farías CE, Amaral MM, Kempfer AC, Votta R, et al. (2004) von Willebrand factor-cleaving protease (ADAMTS13) activity in normal non-pregnant women, pregnant and post-delivery women. Thromb Haemost 92: 1320-1326.

18. Mannucci PM, Canciani MT, Forza I, Lussana F, Lattuada A, et al. (2001) Changes in health and disease of the metalloprotease that cleaves von Willebrand factor. Blood 98: 2730-2735.

19. Bernardo A, Ball C, Nolasco L, Moake JF, Dong JF (2004) Effects of inflammatory cytokines on the release and cleavage of the endothelial cell-derived ultralarge von Willebrand factor multimers under flow. Blood 104: 100-106.

20. Moake JL (2007) Thrombotic thrombocytopenic purpura. In: Kithens CS, Alving $\mathrm{BM}$, Kessler CM, (eds.). Consultative hemostasis and thrombosis. (2ndedn), Saunders Elsevier, Philadelphia, USA. Pg: 405-417.

21. Arya M, Anvari B, Romo GM, Cruz MA, Dong JF, et al. (2002) Ultralarge multimers of von Willebrand factor form spontaneous high-strength bonds with the platelet glycoprotein Ib-IX complex: studies using optical tweezers. Blood 99: 3971-3977.

22. Moake JL, McPherson PD (1989) Abnormalities of von Willebrand factor multimers in thrombotic thrombocytopenic purpura and the hemolytic-uremic syndrome. Am J Med 87: 9-15.
23. Levy GG, Nichols WC, Lian EC, Foroud T, McClintick JN, et al. (2001) Mutations in a member of the ADAMTS gene family cause thrombotic thrombocytopenic purpura. Nature 413: 488-494.

24. George JN (2003) The association of pregnancy with thrombotic thrombocytopenic purpura-hemolytic uremic syndrome. Curr Opin Hematol 10: 339344

25. Vesely SK, George JN, Lämmle B, Studt JD, Alberio L, et al. (2003) ADAMTS13 activity in thrombotic thrombocytopenic purpura-hemolytic uremic syndrome: relation to presenting features and clinical outcomes in a prospective cohort of 142 patients. Blood 102: 60-68.

26. Lattuada A, Rossi E, Calzarossa C, Candolfi R, Mannucci PM (2003) Mild to moderate reduction of a von Willebrand factor cleaving protease (ADAMTS-13) in pregnant women with HELLP microangiopathic syndrome. Haematologica 88: 1029-1034.

27. Austin SK, Starke RD, Lawrie AS, Cohen H, Machin SJ, et al. (2008) The VWF/ADAMTS13 axis in the antiphospholipid syndrome: ADAMTS13 antibodies and ADAMTS13 dysfunction. Br J Haematol 141: 536-544.

28. Aref S, Goda H (2013) Increased VWF antigen levels and decreased ADAMTS13 activity in preeclampsia. Hematology 18: 237-241.

29. Holmes VA, Wallace JM, Alexander HD, Gilmore WS, Bradbury I, et al (2005) Homocysteine is lower in the third trimester of pregnancy in women with enhanced folate status from continued folic acid supplementation. Clin Chem 51: 629-634.

30. Walker MC, Smith GN, Perkins SL, Keely EJ, Garner PR (1999) Changes in homocysteine levels during normal pregnancy. Am J Obstet Gynecol 180: 660-664.

31. Holmes VA, Wallace JM, Gilmore WS, McFaul P, Alexander HD (2002) Tissue factor expression on monocyte subpopulations during normal pregnancy. Thromb Haemost 87: 953-958.

32. Hellgren M (2003) Hemostasis during normal pregnancy and puerperium Semin Thromb Hemost 29: 125-130.

33. Kemkes-Matthes B (2001) [Changes in the blood coagulation system in pregnancy]. Z Kardiol 90: 45-48.

34. Uchikova EH, Ledjev II (2005) Changes in haemostasis during normal pregnancy. Eur J Obstet Gynecol Reprod Biol 119: 185-188.

35. Bonnar J (1978) Hemostatic function and coagulopathy during pregnancy. Obstet Gynecol Annu 7: 195-217.

36. Bremme KA (2003) Haemostatic changes in pregnancy. Best Pract Res Clin Haematol 16: 153-168.

37. Persson BL, Stenberg P, Holmberg L, Astedt B (1980) Transamidating enzymes in maternal plasma and placenta in human pregnancies complicated by intrauterine growth retardation. J Dev Physiol 2: 37-46.

38. Othman M, Falcón BJ, Kadir R (2010) Global hemostasis in pregnancy: are we using thromboelastography to its full potential? Semin Thromb Hemost 36: $738-746$

39. Prisco D, Cluti G, Falciani M (2005) Hemostatic changes in normal pregnancy. Haematologica reports I: 1-5.

40. Ramsay M (2010) Normal hematological changes during pregnancy and the puerperium. In: Pavord S, Hunt B (eds.). The Obstetric Hematology Manual. Cambridge University Press, New York, USA. Pg: 3-12.

41. Thornton P, Douglas J (2010) Coagulation in pregnancy. Best Pract Res Clin Obstet Gynaecol 24: 339-352.

42. Carson SD, Ramsey CA (1985) Tissue factor (coagulation factor III) is present in placental microvilli and cofractionates with microvilli membrane proteins. Placenta 6: 5-12.

43. Uszyński M, Uszyński W (2011) Coagulation and fibrinolysis in amniotic fluid: physiology and observations on amniotic fluid embolism, preterm fetal membrane rupture, and pre-eclampsia. Semin Thromb Hemost 37: 165-174. 
Citation: Momot AP, Semenova NA, Belozerov DE, Trukhina DA, Kudinova IY (2016) The Dynamics of the Hemostatic Parameters in Physiological Pregnancy and After Delivery. J Hematol Blood Transfus Disord 3: 005

44. Uszyński M, Zekanowska E, Uszyński W, Kuczyński J (2001) Tissue Factor (TF) and Tissue Factor Pathway Inhibitor (TFPI) in amniotic fluid and blood plasma: implications for the mechanism of amniotic fluid embolism. Eur $J$ Obstet Gynecol Reprod Biol 95: 163-166.

45. Erez O, Espinoza J, Chaiworapongsa T, Gotsch F, Kusanovic JP, et al (2008) A link between a hemostatic disorder and preterm PROM: a role for tissue factor and tissue factor pathway inhibitor. J Matern Fetal Neonatal Med 21: 732-744.

46. Erez O, Romero R, Hoppensteadt D, Than NG, Fareed J, et al. (2008) Tissue factor and its natural inhibitor in pre-eclampsia and SGA. J Matern Fetal Neonatal Med 21: 855-869.

47. Erez O, Romero R, Vaisbuch E, Kusanovic JP, Mazaki-Tovi S, et al. (2010) High tissue factor activity and low tissue factor pathway inhibitor concentrations in patients with preterm labor. J Matern Fetal Neonatal Med 23: 23-33.

48. Dargaud Y, Hierso S, Rugeri L, Battie C, Gaucherand P, et al. (2010) Endogenous thrombin potential, prothrombin fragment $1+2$ and D-dimers during pregnancy. Thromb Haemost 103: 469-471.

49. Rosenkranz A, Hiden M, Leschnik B, Weiss EC, Schlembach D, et al. (2008) Calibrated automated thrombin generation in normal uncomplicated pregnancy. Thromb Haemost 99: 331-337.

50. Segers O, van Oerle Rv, ten Cate Ht, Rosing J, Castoldi E (2010) Thrombin generation as an intermediate phenotype for venous thrombosis. Thromb Haemost 103: 114-122.

51. van Veen JJ, Gatt A, Makris M (2008) Thrombin generation testing in routine clinical practice: are we there yet? $\mathrm{Br} \mathrm{J}$ Haematol 142: 889-903.

52. Eichinger S (2005) D-dimer testing in pregnancy. Semin Vasc Med 5: 375378.

53. Kline JA, Williams GW, Hernandez-Nino J (2005) D-dimer concentrations in normal pregnancy: new diagnostic thresholds are needed. Clin Chem 51: 825-829.

54. Toh $\mathrm{CH}$, Hoots WK, SSC on Disseminated Intravascular Coagulation of the ISTH (2007) The scoring system of the Scientific and Standardisation Committee on Disseminated Intravascular Coagulation of the International Society on Thrombosis and Haemostasis: a 5-year overview. J Thromb Haemost 5: 604-606.

55. Voves C, Wuillemin WA, Zeerleder S (2006) International Society on Thrombosis and Haemostasis score for overt disseminated intravascular coagulation predicts organ dysfunction and fatality in sepsis patients. Blood Coagul Fibrinolysis 17: 445-451.

56. Wells PS, Anderson DR, Rodger M, Forgie M, Kearon C, et al. (2003) Evaluation of D-dimer in the diagnosis of suspected deep-vein thrombosis. N Engl J Med 349: 1227-1235.

57. Chan WS, Chunilal S, Lee A, Crowther M, Rodger M, et al. (2007) A red blood cell agglutination D-dimer test to exclude deep venous thrombosis in pregnancy. Ann Intern Med 147: 165-170.

58. Onishi H, Kaniyu K, Iwashita M, Tanaka A, Watanabe T (2007) Fibrin monomer complex in normal pregnant women: a potential thrombotic marker in pregnancy. Ann Clin Biochem 44: 449-454.

59. Kovac M, Mikovic Z, Rakicevic L, Srzentic S, Mandic V, et al. (2010) The use of $D$-dimer with new cutoff can be useful in diagnosis of venous thromboembolism in pregnancy. Eur J Obstet Gynecol Reprod Biol 148: 27-30.

60. Sarig G, Blumenfeld Z, Leiba R, Lanir N, Brenner B (2005) Modulation of systemic hemostatic parameters by enoxaparin during gestation in women with thrombophilia and pregnancy loss. Thromb Haemost 94: 980-985.

61. O'Riordan MN, Higgins JR (2003) Haemostasis in normal and abnormal pregnancy. Best Pract Res Clin Obstet Gynaecol 17: 385-396.

62. Cerneca K, Ricci G, Simeone R, Malisano M, Alberico S, et al. (1997) Coagulation and fibrinolysis changes in normal pregnancy. Increased levels of procoagulants and reduced levels of inhibitors during pregnancy induce a hypercoagulable state, combined with a reactive fibrinolysis. Eur J Obstet Gynecol Reprod Biol 73: 31-36.
63. Clark P, Brennand J, Conkie JA, McCall F, Greer IA, et al. (1998) Activated protein $\mathrm{C}$ sensitivity, protein $\mathrm{C}$, protein $\mathrm{S}$ and coagulation in normal pregnancy. Thromb Haemost 79: 1166-1170.

64. Kjellberg U, Andersson NE, Rosén S, Tengborn L, Hellgren M (1999) APC resistance and other haemostatic variables during pregnancy and puerperium. Thromb Haemost 81: 527-531.

65. Boffa MC, Valsecchi L, Fausto A, Gozin D, Vigano'D'Angelo S, et al. (1998) Predictive value of plasma thrombomodulin in preeclampsia and gestational hypertension. Thromb Haemost 79: 1092-1095.

66. de Moerloose P, Mermillod N, Amiral J, Reber G (1998) Thrombomodulin levels during normal pregnancy, at delivery and in the postpartum: comparison with tissue-type plasminogen activator and plasminogen activator inhibitor-1. Thromb Haemost 79: 554-556.

67. Howie PW (1979) Blood clotting and fibrinolysis in pregnancy. Postgrad Med J 55: 362-366.

68. Mousa HA, Downey C, Alfirevic Z, Toh CH (2004) Thrombin activatable fibrinolysis inhibitor and its fibrinolytic effect in normal pregnancy. Thromb Haemost 92: 1025-1031.

69. Cadroy Y, Grandjean H, Pichon J, Desprats R, Berrebi A, et al. (1993) Evaluation of six markers of haemostatic system in normal pregnancy and pregnancy complicated by hypertension or pre-eclampsia. Br J Obstet Gynaecol 100: 416-420.

70. Ishii A, Yamada S, Yamada R, Hamada H (1994) t-PA activity in peripheral blood obtained from pregnant women. J Perinat Med 22: 113-117.

71. Kruithof EK, Tran-Thang C, Gudinchet A, Hauert J, Nicoloso G, et al. (1987) Fibrinolysis in pregnancy: a study of plasminogen activator inhibitors. Blood 69: 460-466.

72. Wright JG, Cooper P, Astedt B, Lecander I, Wilde JT, et al. (1988) Fibrinolysis during normal human pregnancy: complex inter-relationships between plasma levels of tissue plasminogen activator and inhibitors and the euglobulin clot lysis time. Br J Haematol 69: 253-258.

73. Paniccia R, Prisco D, Bandinelli B, Fedi S, Giusti B, et al. (2002) Plasma and serum levels of D-dimer and their correlations with other hemostatic parameters in pregnancy. Thromb Res 105: 257-262.

74. Stirling Y, Woolf L, North WR, Seghatchian MJ, Meade TW (1984) Haemostasis in normal pregnancy. Thromb Haemost 52: 176-182.

75. Blombäck M (1991) Changes in blood coagulation and fibrinolysis during pregnancy and the menstrual cycle: new methods for detecting the changes. Adv Contracept 7: 259 -272.

76. Borrelli AL, De Lucia D, Bernacchi M, Napolitano M, Di Domenico A, et al. (2006) [Haemocoagulative modifications correlated with pregnancy]. Minerva Ginecol 58: 315-322.

77. Choi JW, Pai SH (2002) Tissue plasminogen activator levels change with plasma fibrinogen concentrations during pregnancy. Ann Hematol 81: 611615

78. Meltzer ME, Lisman T, de Groot PG, Meijers JC, le Cessie S, et al. (2010) Venous thrombosis risk associated with plasma hypofibrinolysis is explained by elevated plasma levels of TAFI and PAI-1. Blood 116: 113-121.

79. Maiello M, Torella M, Caserta L, Caserta R, Sessa M, et al. (2006) [Hypercoagulability during pregnancy: evidences for a thrombophilic state]. Minerva Ginecol 58: 417-422.

80. Stephens RW, Pöllänen J, Tapiovaara H, Leung KC, Sim PS, et al. (1989) Activation of pro-urokinase and plasminogen on human sarcoma cells: a proteolytic system with surface-bound reactants. J Cell Biol 108: 1987-1995.

81. Saleh Y, Pawelec M, Siewinski M, Karmowski A, Sebzda T (2004) Expression of urokinase plasminogen activator, its receptor and plasminogen activator inhibitor in the plasma of pathological pregnancy women. Intern $\mathrm{J}$ Gynecol Obstetrics 5 
Citation: Momot AP, Semenova NA, Belozerov DE, Trukhina DA, Kudinova IY (2016) The Dynamics of the Hemostatic Parameters in Physiological Pregnancy and After Delivery. J Hematol Blood Transfus Disord 3: 005

82. Koh SC, Anandakumar C, Biswas A (1999) Coagulation and fibrinolysis in viable mid-trimester pregnancies of normal, intrauterine growth retardation, chromosomal anomalies and hydrops fetalis and their eventual obstetric outcome. J Perinat Med 27: 458-464.

83. Lindoff C, Astedt B (1994) Plasminogen activator of urokinase type and its inhibitor of placental type in hypertensive pregnancies and in intrauterine growth retardation: possible markers of placental function. Am J Obstet Gynecol 171: 60-64.

84. Booth NA, MacGregor IR, Hunter NR, Bennett B (1987) Plasminogen activator inhibitor from human endothelial cells. Purification and partial characterization. Eur J Biochem 165: 595-600.

85. Schiffman FJ (2001) Hematologic patophysiology. Lippincott-Renal Publishens, Philadelphia, New York, USA Pg: 201

86. Estellés A, Gilabert J, Espana F, Aznar J, Gómez-Lechón MJ (1987) Fibrinolysis in preeclampsia. Fibrinolysis 1: 209-214.

87. Astedt B, Lindoff C, Lecander I (1998) Significance of the Plasminogen Activator Inhibitor of placental type (PAI-2) in pregnancy. Semin Thromb Hemost 24: 431-435.

88. Redlitz A, Tan AK, Eaton DL, Plow EF (1995) Plasma carboxypeptidases as regulators of the plasminogen system. J Clin Invest 96: 2534-2538.

89. Wang W, Boffa MB, Bajzar L, Walker JB, Nesheim ME (1998) A study of the mechanism of inhibition of fibrinolysis by activated thrombin-activable fibrinolysis inhibitor. J Biol Chem 273: 27176-27181.

90. Ku DH, Arkel YS, Paidas MP, Lockwood CJ (2003) Circulating levels of Inflammatory cytokines (IL-1 beta and TNF-alpha), resistance to activated protein $\mathrm{C}$, thrombin and fibrin generation in uncomplicated pregnancies. Thromb Haemost 90: 1074-1079.

91. Bouma BN, Meijers JC (2004) New insights into factors affecting clot stability: A role for Thrombin Activatable Fibrinolysis Inhibitor (TAFI; plasma procarboxy peptidase $B$, plasma procarboxypeptidase $U$, procarboxypeptidase R). Semin Hematol 41: 13-19.
92. Al-Horani RA (2014) Serpin regulation of fibrinolytic system: implications for therapeutic applications in cardiovascular diseases. Cardiovasc Hematol Agents Med Chem 12: 91-125.

93. Lee KN, Jackson KW, Christiansen VJ, Chung KH, McKee PA (2004) Alpha2-antiplasmin: potential therapeutic roles in fibrin survival and removal. Curr Med Chem Cardiovasc Hematol Agents 2: 303-310.

94. Aoki N (2005) The past, present and future of plasmin inhibitor. Thromb Res 116: $455-464$

95. Coughlin PB (2005) Antiplasmin: the forgotten serpin? FEBS J 272: 48524857.

96. Schaller J, Gerber SS (2011) The plasmin-antiplasmin system: structural and functional aspects. Cell Mol Life Sci 68: 785-801.

97. Carpenter SL, Mathew $P$ (2008) Alpha2-antiplasmin and its deficiency: fibrinolysis out of balance. Haemophilia 14: 1250-1254

98. Meesters EW, Hansen H, Spronk HM, Hamulyak K, Rosing J, et al. (2007) The inflammation and coagulation cross-talk in patients with systemic lupus erythematosus. Blood Coagul Fibrinolysis 18: 21-28.

99. Brenner B, Francis CW, Totterman S, Kessler CM, Rao AK, et al. (1990) Quantitation of venous clot lysis with the D-dimer immunoassay during fibrinolytic therapy requires correction for soluble fibrin degradation. Circulation 81: $1818-1825$

100.Feddersen C (1983) Proteolytic degradation products from fibrinogen and fibrin with special respect to non-covalently associated complexes. Influence of fibrin crosslinking. Lysability and other properties. Scand J Clin Lab Invest Suppl 166: 1-60.

101.Selmayr E, Müller-Berghaus G (1985) Soluble crosslinked fibrin(ogen) polymers. Thromb Haemost 54: 804-807.

102. Momot A, Lydina I, Tsyvkina L, Borisova O, Serduk G (2012) The Means of Progress in Improving the Results of in vitro Fertilization Based on the Identification and Correction of the Pathology of Hemostasis. In: Darwish AMM (eds.). Enhancing Success of Assisted Reproduction. InTech, Rijeka, Croatia. 